\title{
The Impact of Government Subsidy on Supply Chains’ Sustainability Innovation
}

\author{
Jen-Yi Chen (Jay) \\ Monte Ahuja College of Business, Cleveland State University \\ Stanko Dimitrov \\ Department of Management Sciences, University of Waterloo \\ Hubert Pun \\ Ivey Business School, Western University Canada
}

Motivated by recently observed industry and government practices, in this paper we endogenize government subsidy in a research joint venture (RJV). In particular, we present a three-player game in which a government determines the amount of subsidy for a supply chain consisting of a manufacturer and a retailer conducting an RJV on a sustainable product. In addition to the retail and wholesale pricing decisions, the firms must determine the level of innovation effort as well as the division of innovation costs between the partners. In our analysis we consider two forms of RJV formation (retailer and manufacturer initiated) and two types of subsidy (per-unit production subsidy and innovation effort subsidy). We find that: (a) the government should never use both types of subsidies simultaneously for any cost-reduction research and development (R\&D) effort, (b) whenever effort subsidy is present the government is indifferent as to how the RJV is formed, and (c) even though firms benefit most from initiating the RJV in many cases, the manufacturer is worse off by initiating the RJV if R\&D effort increases per-unit production cost, innovation or production cost is high and only per-unit production subsidy is present.

Keywords: supply chain management; government subsidy; innovation; sustainability

\section{Introduction}

Many governments throughout the world have established clear sustainability policies on sustainable development, resource use, and energy efficiency (Canada, 2008; NSW, 2014; Obama, 2015). In some cases, governments have incentivized industrial firms in order to meet these sustainability policies (Espinoza, 2012). Government incentives may take various forms 
(Global Subsidies Initiative, 2015). The two major ways producers benefit from government subsidy are through a direct cost from the government (e.g., grants and tax concessions) or through the market (e.g., a domestic government taxing foreign-made products so heavily that they are not competitive with domestic products).

In practice a government may offer multiple subsidies at the same time, for example tax foreign-made products while at the same time providing grants to the same industries to create new products. These multiple subsidies may have confounding effects. For example, taxing market substitutes may disincentivize new product creation (competition may not be needed as competitors' goods have an effectively higher price), while grants may actually incentivize new product creation as innovation is now cheaper. In this paper, we will focus on grant subsidies to better understand complexities associated with different types of such subsidies. As market subsidies are needed only in the presence of competing products, we do not consider competing products in this work. We note that grant subsidy may also take a variety of forms. First, subsidy may be a function of the total innovation investment made - what we refer to as innovation effort subsidy. For example, to encourage sustainable innovation, part of the American Recovery and Reinvestment Act of 2009 allocated \$400 million to Advanced Research Projects AgencyEnergy to fund research and development (R\&D) of energy technologies (U.S. Congress, 2009). Alternatively, subsidy may be a function of the total amount produced; we refer to this subsidy as per-unit production subsidy. To illustrate, the Government of India Ministry of Power, through the UJALA - Unnat Jyoti By Affordable LEDs for All program, subsidized the procurement of LED lightbulbs in the country (Ministry of Power, 2017).

There are also cases in which the government uses both innovation effort and per-unit production subsidy, such as the Energy Policy Act of 2005 (U.S. Congress, 2005) see sections 
208 (sugar cane ethanol program), section 609 (rural and remote communities electrification grants), and section 202 (renewable energy production incentive). The United States of America and India are not the only countries to consider subsidies. For example, United Kingdom's innovation agency, Innovate UK, has a series of grants to support collaboration amongst various entities to foster sustainable innovation, along with other forms of innovation (InnovateUK, 2015).

In addition to governments identifying sustainability as a policy component, consumers are willing to pay more for products that are perceived as better for the environment (Nielsen, 2014)—referred to as sustainable products. In order to appeal to such demands, retailers and manufacturers are producing more sustainable goods, such as Clorox's line of natural cleaning products, Green Works (Korn, 2008). However, even with government subsidy, sustainability, sustainable products, and environmentally friendly business practices, are growing business challenges as the costs of research and development and manufacturing may be prohibitive for any one firm alone. Accordingly, manufacturers and retailers collaborate by spreading costs of developing sustainable practices and product via RJVs to make sustainable products.

In fact, a recent survey of Italian Automotive executives (Rossini, Ciarapica, Matt, \& Spena, 2016) noted that firms that needed to innovate, in the form of a radical supply chain change, to accommodate electric vehicles, are considering entering into RJVs at a higher rate than those that do not need to innovate. In addition, a worldwide survey ${ }^{1}$ of executives and managers by MIT Sloan Management Review (SMR) found that 90\% of the 2,587 respondents "Agreed or Somewhat Agreed" that collaborations are needed for sustainability (Kiron, Kruschwitz, Haanaes, \& Reeves, 2015). Those same respondents stated that $47 \%$ of their firms

\footnotetext{
${ }^{1}$ In collaboration with The Boston Consulting Group and the United Nations Global Compact
} 
are currently engaged in sustainability-related collaborations, with over $50 \%$ of these collaborations are classified as very or quite successful. The main reason firms engage in sustainability, as found by the MIT SMR survey is to increase their reputation and build their brand, followed closely by product and service innovation as well as expanding into new markets.

In addition to the various examples provided by Kiron, Kruschwitz, Haanaes, \& Reeves (2015), a few specific examples are available; Walmart, for instances, recently worked with its manufacturers to reduce the volume of water in laundry detergents, and with Clorox to safely concentrate bleach (Baker, 2014). Similarly, AltEn, the world’s largest solar-boat manufacturer, formed a joint venture with an Indian firm to make solar boats for the Indian market in which the firm procures its critical power components from AltEn. In addition, 33\% of solar ferry costs are subsidies by the Indian Ministry of New and Renewable Energy (Srinivasan, 2016). Though the intents are the same, the dynamics of these RJVs may differ; the retailer may initiate the partnership, as in the case of Walmart, or the manufacturer, as in the case of AltEn. We note that through a long-term RJV, Sematech, firms such as Advanced Micro Devices (AMD) find that they get larger returns than they expected (Office, 1992) while developing next generation products for current and new market segments. We reiterate that the main goal of our paper is to study how different RJV formation dynamics and subsidy affect sustainable innovations. To isolate the desired effects, we do not consider competing product in this study that may be generated as a result of a RJV.

It remains unclear exactly how all of these components fit together in determining research efforts, prices, and government subsidy, and this paper considers the following factors: government incentives to promote sustainable development and supply chain partners working 
together in RJVs to make sustainable products. We consider a manufacturer and a retailer selling products to consumers. In addition to the wholesale and retail pricing decisions, the supply chain partners must decide the level of sustainability effort exerted and each partner's investment responsibilities. Either of the two firms may initiate the collaboration, with the initiating firm setting how innovation effort costs are distributed among the collaborating firms. In particular, when the retailer initiates collaboration (e.g., Walmart working with Clorox), the retailer motivates the manufacturer to exert effort by proposing the amount of innovation cost that it would be responsible for, and then the manufacturer decides the level of innovation effort. On the other hand, when the manufacturer initiates collaboration (e.g., the AltEn example), the manufacturer determines both the collaboration level and the effort level. Though the two surveys (Kiron et al., 2015; Rossini et al., 2016) clearly identified that collaborations are needed and desired by firms interested in sustainability; the exact formation of the collaboration, which party initiates the venture, is not always clearly known by a third-party observer (only the final RJV is observed and rarely are RJV dynamics discussed in the literature) and thus we consider both formation dynamics. To motivate the supply chain to innovate, the government provides per-unit production subsidy and/or innovation investment subsidy. Specifically, we aim to glean managerial insights by exploring the following research questions:

- What is the impact of different types of subsidy (per-unit production versus innovation effort) on subsidy parameters, collaboration and effort levels, and firms' profits?

- What is the impact of collaboration dynamics (retailer-initiated versus manufacturerinitiated RJVs) on subsidy parameters, collaboration and effort levels, and firms' profits? The first important message of our paper is that, when it comes to cost-reducing R\&D effort, the government should never use both types of subsidy simultaneously. When R\&D effort 
instead increases production cost, regardless of which firm initiates the collaboration, we find that the government can best incentivize the supply chain to collaborate at the desired level and exert the most innovation effort by leveraging both forms of subsidy; the total subsidy required when offering both form of subsidy is smaller than if the government used either subsidy by itself. Furthermore, in contrast to the government's preference to offer both types of subsidy, manufacturers and retailers are better off if only per-unit subsidy is offered, and are worse off if only innovation effort subsidy is offered.

In light of first-mover advantage, one might expect that firms to prefer initiating rather than following in the collaboration process. Interestingly, our results show that this intuition does not always hold. In particular, when the government uses per-unit production subsidy alone, the manufacturer may be worse off initiating the collaboration when innovation or production is not costly. Moreover, we find that the government is indifferent to the collaboration dynamics when it provides innovation subsidy, as it induces the same innovation effort, demand, and price.

These insights will help policy makers and business leaders find win-win solutions when pursuing sustainability in response to societal ills (as is the case for governments), or in response to consumer demand (as is the case for supply chain partners). Further managerial insights that may be gleaned from our results are discussed in the concluding section.

In the remainder of the paper, we first outline the literature related to our work, following which, we present our model and analysis. We then discuss our findings and key insights before offering our conclusions and suggestions for future research.

\section{Literature Review}


Our research is related to three streams of literature in both the operations management (OM) and economics literature as it concerns social welfare and government policy, and collaborative innovation in sustainable operations.

The area of sustainable operations is of growing interest in the OM field (Drake \& Spinler, 2013). For example, Drake et al. (2015) study the impact of tax and cap-and-trade regulations on a firm's technology and capacity decisions. Girotra \& Netessine (2013) provide a business model framework for firm sustainability and identify four significant contexts to consider when creating/augmenting a business model. Considering the effects of environmental innovations on both production cost and demand at different stages of the product life cycle, Raz et al. (2013) study a newsvendor, determining its innovation efforts at each stage in addition to production quantity. However, most literature in this area focuses on single firms' decisions or does not consider government subsidy (see Tang and Zhou (2012) for a review of earlier works) while our research incorporates collaborative efforts between two vertical firms when studying government’s optimal subsidy policies.

Within the field of sustainability, one stream close to our work is research studying social welfare and regulation. For example, in the context of a closed-loop supply chain, Atasu et al. (2013) and many of the references contained therein (e.g., Palmer and Walls (1997) from the perspective of environmental economics and Ozdemir et al. (2012) from OM perspective) study how the government should design take-back or recycling legislation when incorporating externalities of the operations to maximize social welfare. Krass et al. (2013) and Raz and Ovchinnikov (2015) consider the use of a government rebate and/or subsidy for firms producing public interest goods (goods with externality, for example, through emissions-reducing technologies) and analyze the government's ability to coordinate this system. From the 
perspective of a non-governmental organization, Kraft et al. (2013) assess whether it is more effective to indirectly target the regulatory body or directly target the industry in order to influence firms to remove environmentally hazardous substances. Cohen et al. (2016) study how demand uncertainty impacts green technology adoption and a manufacturer's production and pricing decisions when designing per-unit government subsidies directly to end consumers. To further understand the effect of government subsidy policies on vertical collaboration (see also Popp et al. (2009) for a detailed review of earlier works in the field of environmental economics), our research specifically considers two distinct subsidy types, upfront innovation cost and post-innovation per-unit production cost.

Our study is also closely related to the area of collaborative innovation. Krishnan and Ulrich (2001) and Krishnan and Loch (2005) provide reviews of the literature regarding product development and innovation. However, as observed by Ge et al. (2014), most works in this literature focused on horizontal R\&D cooperation (Bhaskaran \& Krishnan, 2009; Ge \& Hu, 2008) (please see Veugelers (1998) for a review of earlier works). Few papers investigate the collaboration of firms with vertical relations. Talluri et al. (2010) consider a manufacturer allocating funds to its suppliers to improve their performance and find scenarios in which manufacturers benefit from collaborating with their suppliers and even amongst themselves in improving the performance of a joint supplier. Kim and Netessine (2013) investigate the joint effect of asymmetric information and procurement contracting strategies on the collaborative effort between supply chain parties, but their focus on collaboration primarily addresses agency issues. The research of Ghosh and Shah (2015) is most related to our work in exploring collaboration between supply chain players; however, while they consider a retailer-driven and a restrictive bargaining game between two firms of equal power, we examine two representative 
cases in which either the manufacturer or the retailer can initiate collaboration as illustrated in the introductory examples to study the effect of relative power in defining contract terms between two vertical firms as illustrated in the introduction. Furthermore, we expand on Ghosh and Shah (2015) by also contemplating situations in which innovation effort impacts production cost. The most distinct difference, however, is while Ghosh and Shah (2015) focus on studying the impact of a cost-sharing mechanism from a supply chain perspective (only consider a supply chain, retailer and manufacturer), in this paper we shed light into how government subsidy programs may be designed to achieve socially best outcome (we consider a supply chain, retailer and manufacturer, and a government) with focus on sustainable innovation. To do so, we first explore how the relative power of the two supply chain parties of vertical relation affects the collaboration. With knowledge of such market dynamics, we then investigate how government policy influences the collaborative innovation between these two supply chain entities, which directly affect the environment and society.

To summarize, our research contributes to the literature by analyzing the government incentives for a supply chain to collaborate on sustainable innovation (Ghosh \& Shah, 2015) that benefits not only the supply chain parties and consumers but also the environment and society. To the best of our knowledge, this is the first model jointly considering two types of incentives (innovation and production subsidies) and two types of supply chain collaboration (one led by the upstream manufacturer and the other led by the downstream retailer). In addition, this paper incorporates two themes, production technology choice (relating to environmental performance) and forward (multi-echelon) supply chain, among five active themes proposed by Drake and Spinler (2013). It also addresses two of the four research gaps, the largely ignored market forces and vertically aligned sustainable strategies, identified by Tang and Zhou (2012). 


\section{The Model}

Consider a manufacturer and a retailer that jointly develop a sustainable product. The retailer sells the product to customers at a retail price, $p$, and the manufacturer exerts sustainability effort, $e$, and this sustainability effort increases demand. For example, customers may have higher utility consuming the new sustainable product, so their willingness-to-pay increases, leading to greater demand. In particular, the demand for the product is:

$$
D=1+e-p
$$

Note that without loss of generality, like $p$, we assume $e$ has a leading coefficient of one (effort sensitivity of demand $\gamma=1$ ) since the results for arbitrary $\gamma$ can be obtained through a scaling of the equilibrium effort $e^{\prime}=\frac{e}{\gamma}$ and corresponding adjustments of other effort-related coefficients.

To capture the decreasing marginal effect of effort, we assume that the effort cost is of the quadratic form, $k e^{2}$, where $k>0$ is the coefficient of the effort cost, similar to Arora and Ceccagnoli (2006), and Atasu and Subramanian (2012). We assume the sustainability effort may also affect the per-unit production cost, $c=c_{0}+\beta e$, with the base cost, $c_{0}$, normalized to zero for no effort exerted, where $\beta>0$ represents the scenario in which sustainability effort leads to an increase in per-unit production cost. For example, environmentally friendly production processes increase the cost of coffee production by about 30\% (Kojoyan, 2014). We will formally define and discuss the bounds on $\beta$ in more detail later, but in order for the manufacturer to exert positive effort, we further restrict our attention to $0<\beta<1$. Similarly, throughout our analysis we will find that there are various bounds on $k$, that are functions of other exogenous model parameters, which lead to switching in the equilibrium decisions. 
In order to encourage sustainability innovation, the government provides subsidy, collectively referred to as $\Phi$, to the two firms, as discussed in the introductory section. For example, the government can provide a tax credit to the firms for each unit produced, i.e., $\Phi=$ $t D$, where $t \geq 0$ is the per-unit tax credit for each produced unit. Note that we are assuming market-clearing prices; as such, all units produced are sold. The government might also encourage innovation by subsidizing the cost of innovation, i.e., $\Phi=\Delta k e^{2}$, where $0 \leq \Delta k<k$, so the effective cost of innovation reduces to $(k-\Delta k) e^{2}$. The sustainability effort cost and the government subsidy are shared between the two firms with $\lambda$ fraction going to the manufacturer and the remaining amount, $1-\lambda$, to the retailer. In the case when both forms of subsidy is offered, $\Phi=t D+\Delta k e^{2}$.

The unit payment between the two firms is $w$, which is commonly known as the wholesale price when the payment is from the retailer to the manufacturer, and is the transfer payment when the payment is from the manufacturer to the retailer. Given the notation set forth in this section, and assuming the only profit the firms and government derive are from the considered setting, the profit functions of the manufacturer and the retailer are as follows:

$$
\begin{gathered}
\pi_{M}=(w-\beta e) D-\lambda k e^{2}+\lambda \Phi \\
\pi_{R}=(p-w) D-(1-\lambda) k e^{2}+(1-\lambda) \Phi .
\end{gathered}
$$

By incorporating positive externality from sustainability innovation, subsidy, firms’ profits, and consumer surplus, as commonly defined in the literature (Krass et al., 2013; Raz \& Ovchinnikov, 2015), the government's objective function can be expressed as follows:

$$
\pi_{G}=\alpha e-\Phi+\pi_{M}+\pi_{R}+\frac{D^{2}}{2}
$$

The first term corresponds to the external benefits of sustainability innovation effort $(\alpha>$ 0 is a constant marginal benefit in effort level). Most literature on sustainability (Atasu, Van 
Wassenhove, \& Sarvary, 2009; Raz \& Ovchinnikov, 2015) assumes constant marginal benefit in quantity unit, i.e., $\alpha D$, as the authors focus on the deployment (units sold) of an exogenously given sustainable technology, i.e., a predetermined effort level $e>0$ and thus a constant $\alpha e>0$ which can be left out from the objective function. In contrast, our paper focuses on determining the optimal effort level in developing sustainability innovation, i.e., an endogenous effort level $e \geq 0$ and thus an indispensable sustainability benefit term $\alpha e$. Therefore, motivated by the work of Atasu et al. (2009), and Raz and Ovchinnikov (2015), we use the same linear government benefit from the sustainable product, but consider only the direct sustainability benefit through innovation effort, $e$. In particular, if there is no effort exerted in equilibrium, then there is no government benefit even if many units are sold, as these are not sustainable products which is explicitly captured in our model $(\alpha e=0<\alpha D)$. The second term in $\pi_{G}$ is the total subsidy provided to the two firms, and the remaining terms are the social aspect, which includes the profit of the two firms and the consumer surplus. As commonly defined in the literature (e.g., Atasu et al. (2009), and Raz and Ovchinnikov (2015)), the consumer surplus is the area of the demand curve below a given price, which can be expressed as $D^{2} / 2$.

Recall that executive level personnel surveys found that that firm-to-firm collaboration is needed by firms interested in sustainability. However, the dynamics of how those collaborations are formed are not explicitly stated. To account for all feasible dynamics, we consider two possible collaboration models between the manufacturer and the retailer. The first is when the retailer initiates collaboration, as in the motivating example of Walmart working with Clorox. Like most of the literature on collaborative innovation (Bhaskaran \& Krishnan, 2009), we assume that the collaboration level is determined prior to innovation effort being made. Therefore, the retailer must motivate the manufacturer to exert effort by sharing the innovation 
cost. In particular, when the retailer initiates collaboration, it first decides the collaboration level, $\lambda$, and then the manufacturer decides the level of effort, $e$. As in Atasu et al. (2009), Krass et al. (2013), and Raz and Ovchinnikov (2015), we assume that the government first decides on the subsidy programs that maximize its objective function, anticipating the manufacturer's and retailer's actions. As a result, the game sequence can be stated as follows:

Step 1: The government decides $\Phi$;

Step 2: The retailer decides $\lambda$;

Step 3: The manufacturer decides $e$ and $w$;

Step 4: The retailer decides $p$.

The second model is when the manufacturer initiates collaboration, as in the AltEn example. In this scenario, the manufacturer determines both the collaboration level, $\lambda$, and the effort level, $e$. The game sequence can be stated as follows:

Step 1: The government decides $\Phi$;

Step 2: The manufacturer decides $\lambda$, $e$, and $w$;

Step 3: The retailer decides $p$.

We solve the game using backward induction, discussed further in the Analysis section. Note that we derive technical restrictions on model parameters when ensuring second-order optimality conditions and taking parameter positivity constraints into consideration.

\section{Analysis}

For ease of exposition, we define system $x y$, where $x \in\{R, M\}$ represents whether the Retailer or the Manufacturer initiates the collaboration, and $y \in\{P, I, B\}$ denotes whether the government provides a Per-unit Production subsidy ( $\Phi=t D)$, an Innovation effort subsidy $\left(\Phi=\Delta k e^{2}\right)$ or 
Both types of subsidy $\left(\Phi=t D+\Delta k e^{2}\right)$. To illustrate, system $R I$ represents the scenario where the retailer initiates RJV collaboration, and the government uses innovation effort subsidy to encourage the firms to innovate.

\subsection{Analytical Results}

As backward induction as the solving procedure is fairly standard, we refer interested readers to Appendix A for the formal analysis but summarize and discuss solutions for two general cases separately: the retailer-initiated collaboration with both types of subsidy $(R B)$, and the manufacturer-initiated collaboration with both types of subsidy $(M B)$. The same appendix also provides the analysis and the equilibrium solutions of the other four cases ( $R P, R I, M P$, and $M I)$.

\begin{tabular}{|l|l|}
\hline Retailer-Initiated RJV & Manufacturer-Initiated RJV \\
\hline$t^{R B}=\frac{3[2 k+\alpha(1-\beta)]}{2 k-(1-\beta)^{2}}$ & $t^{M B}=\frac{3[2 k+\alpha(1-\beta)]}{2 k-(1-\beta)^{2}}$ \\
\hline$\Delta k^{R B}=\frac{16 k \alpha-(9 \alpha+1-\beta)(1-\beta)^{2}}{16(\alpha+1-\beta)}$ & $\Delta k^{M B}=\frac{2 k[2 \alpha-(1-\beta)]-3 \alpha(1-\beta)^{2}}{4(\alpha+1-\beta)}$ \\
\hline$\lambda^{R B}=\frac{8[2 k+\alpha(1-\beta)]}{16 k+(9 \alpha+1-\beta)(1-\beta)}$ & $\lambda^{M B}=\frac{-8 k-(1-\beta)[\alpha-3(1-\beta)]}{3(1-\beta)(\alpha+1-\beta)}$ \\
\hline$e^{R B}=\frac{\alpha+1-\beta}{2 k-(1-\beta)^{2}}$ & $e^{M B}=\frac{\alpha+1-\beta}{2 k-(1-\beta)^{2}}$ \\
\hline$\pi_{M}^{R B}=\frac{[2 k+\alpha(1-\beta)][8 k+(1-\beta)(3 \alpha-(1-\beta))]}{2\left[2 k-(1-\beta)^{2}\right]^{2}}$ & $\pi_{M}^{M B}=\frac{3[2 k+\alpha(1-\beta)][8 k+(1-\beta)(3 \alpha-(1-\beta))]}{4\left[2 k-(1-\beta)^{2}\right]^{2}}$ \\
\hline$\pi_{R}^{R B}=\frac{[8 k+(5 \alpha+(1-\beta))(1-\beta)][8 k+(1-\beta)(3 \alpha-(1-\beta))]}{16\left[2 k-(1-\beta)^{2}\right]^{2}}$ & $\pi_{R}^{M B}=0$ \\
\hline$\pi_{G}^{R B}=\frac{2 k+\alpha[\alpha+2(1-\beta)]}{2\left[2 k-(1-\beta)^{2}\right]}$ & $\pi_{G}^{M B}=\frac{2 k+\alpha[\alpha+2(1-\beta)]}{2\left[2 k-(1-\beta)^{2}\right]}$ \\
\hline
\end{tabular}

Table 1 Equilibrium in Two General RJV Cases

We note that boundary solutions in the general case reduce to special cases that are analyzed separately (in the appendix as the solving procedures are similar), e.g., RP is RB with $\Delta$ $k^{R B}=0$ and $\mathrm{RI}$ is $\mathrm{RB}$ with $t^{R B}=0$. It happens to cost-reducing (negative) $\beta$ cases that interior 
solutions are not feasible since the corresponding retail prices are negative following the same solving procedure. This implies that the government would never use both subsidies simultaneously. For ease of exposition in the main text, we present and discuss only interior solutions that satisfy joint technical conditions for all cases, in particular, $0<\beta<1$ (the bounds are to ensure non-negative solutions and second-order optimality conditions (SOCs)). It is worthnoting that when all technical conditions are met, all three parties' profits are increasing in $\alpha$, suggesting that sustainability innovation can be a win-win strategy for both the government (society) and supply chain partners, if the government (society) benefits significantly from sustainable business practices.

Next we present and discuss our analytical results for all six scenarios considered (four of which are analyzed in Appendix A), two possible collaboration initiators (My v.s. Ry) with three possible subsidy offers $(x P, x I$, and $x B$ ). First, Proposition 1 in Section 4.2 compares the collaboration level and effort $(\lambda, e)$. Then Propositions 2 and 3 in Section 4.3 compare profits of the firms and the government between different types of subsidy and different RJV formation dynamics respectively. To not overcrowd the main insights, we refer interested readers to Appendix B, where we compare the subsidy parameters $(t, \Delta k)$ in Proposition $\mathrm{B} 1$. The proofs of all propositions, those presented in this section and in Appendix B, may be found in Appendix C. In addition, in the same appendix, Lemma 1 shows sensitivity analysis of the decisions ( $t$, $\Delta k, \lambda, e$ ) with respect to the cost of sustainability innovation, $k$, and how innovation changes the per-unit production cost, $\beta$. As we have indicated earlier, besides ensuring that the second order conditions are satisfied, the positivity requirements for the per-unit tax credit $t$, innovation subsidy $\Delta k$, effort $e$, retail price $p$ and demand $D$ must also be satisfied for all of the systems under consideration when we plot figures and discuss results in the main text. 


\subsection{Collaboration Level and Effort Comparisons}

In this subsection, we examine the impact of both retailer-initiated and manufacturer-initiated RJVs on a given type of subsidy (comparisons between systems $R y$ and $M y$ ). Proposition 1 examines these impacts to the collaboration level, $\lambda$, and effort, $e$.

\section{Proposition 1:}

With the innovation cost sufficiently high: the fraction of the sustainability effort cost covered by the manufacturer is always greater under the retailer initiated RJV than the manufacturer initiated RJV. Under the retailer initiated RJV: the fraction of sustainability effort cost covered by the manufacturer is greatest when only per-unit subsidy is present, followed by when both subsidies are present, and finally least when only innovation effort subsidy is present. Under the manufacturer initiated RJV: the fraction of the effort cost covered by the manufacturer reverses.

The innovation effort under the retailer initiated $R J V$ is always weakly dominated by the manufacturer initiated RJV regardless of the subsidy present. The innovation effort is always greatest when both subsidies are present, regardless of the RJV formation dynamics. Innovation effort subsidy results in higher innovation effort than per-unit subsidy, again regardless of the $R J V$ formation dynamics.

Formally, we write the result as:

a. $\lambda^{R y}>\lambda^{M y} ; e^{R P}<e^{M P} \Leftrightarrow k>\Psi_{e 1} ; e^{R I}=e^{M I} ; e^{R B}=e^{M B}$

b. $\lambda^{R P}>\lambda^{R B}>\lambda^{R I} ; e^{R B}>\max \left[e^{R P}, e^{R I}\right] ; e^{R P}<e^{R I} \Leftrightarrow k>\Psi_{e 2}$

c. $\lambda^{M I}>\lambda^{M B}>\lambda^{M P} ; e^{M B}>\max \left[e^{M P}, e^{M I}\right] ; e^{M P}<e^{M I} \Leftrightarrow k>\Psi_{e 3}$ where $\Psi_{e 1}, \Psi_{e 2}$ and $\Psi_{e 3}$ are defined in the proof. 
Proposition 1a shows that for any given type of subsidy, the RJV initiator always sets the collaboration level such that the RJV initiator would have the follower share more of cost than if the follower were the initiator. Moreover, the follower covers the smallest fraction (i.e., $\lambda$ is small when the retailer initiates or $1-\lambda$ is small when the manufacturer initiates) when the government uses innovation subsidy (system $x I$ ) than when the government uses other forms of subsidy (cf. Proposition 1b,c). On the other hand, the follower is responsible for the largest fraction when the government uses per-unit tax credit subsidy (system $x P$ ). In other words, the RJV initiator has the largest share under innovation effort subsidy, but the least share under production subsidy.

With respect to the exerted effort, we first note that the innovation effort is the same regardless of the RJV collaboration dynamic, as long as innovation effort subsidy is present. This is the case because under the $R z, z \in\{I, B\}$, cases, though the retailer passes a larger fraction of the innovation cost to the manufacturer, the government provides larger subsidies to compensate the manufacturer for carrying more of the innovation cost. Further, the innovation effort is greater when the manufacturer initiates the RJV, and only per-unit production subsidy is present and innovation is costly (cf. Proposition 1a). The drivers for the comparison between $e^{R P}$ and $e^{M P}$ are similar to those for the comparison between $t^{R P}$ and $t^{M P}$, and the threshold $\Psi_{e 1}$ is the same as the threshold $\Psi_{1}$ given in Proposition B1a. According to that discussion, when innovation is not costly ( $k$ is small), the fraction of cost/subsidy $(\lambda)$ the manufacturer carries is small, in an absolute sense. As $k$ increases, the value of $\lambda$ becomes more important, and as such the manufacturer would like to keep a larger fraction of the total government subsidy, that is greater under a manufacturer initiated setting than a retailer initiated setting. Therefore, since the 
manufacturer is experiencing a lower net cost from effort, due to retaining a larger fraction of a larger subsidy, the effort exerted by the f per-unit subsidy is present.

We also note that regardless of the RJV structure, the highest effort is always exerted when both forms of subsidy are present (cf. Propositions 1b,c). When only a single type of subsidy is present, the innovation effort is higher when the government uses innovation effort subsidy only if innovation is costly ( $k>\Psi_{e 2}$ for the comparison between $e^{R P}$ and $e^{R I}$, and $k>$ $\Psi_{e 3}$ for the comparison between $e^{M P}$ and $e^{M I}$ ). Effort levels when only a single type of subsidy is present follows from the fact that if the subsidies are fixed, and effort level increases, per-unit subsidy only has a linear benefit for the manufacturer for every unit of effort increase, while innovation effort subsidy has quadratic benefit for every unit of effort increases. The bound is simply the point where the quadratic benefit dominates the linear benefit. Note that the same argument holds when discussing $e^{M P}$ and $e^{M I}$. We present the effort comparison in Figure 1 (retailer-initiated RJV) and in Figure 2 (manufacturer-initiated RJV). We note that the thresholds $\Psi_{e 2}$ and $\Psi_{e 3}$ are functions of $\alpha$ and $\beta$, and these two thresholds decrease in the impact of effort to the per-unit production cost $(\beta)$, meaning that regardless of which firm initiates collaboration, effort is likely to be larger when the government uses innovation subsidy than using per-unit subsidy when sustainability innovation is costly ( $k$ large). Moreover, for a retailer-initiated RJV, we note that as the marginal benefit in effort level to government's objective function $(\alpha)$ increases, both of the grey areas decrease. In addition, for any fixed value of $k, e^{R P}<e^{R I}$ always holds for larger values of $\beta$ than $e^{R P}>e^{R I}$. The boundary between the two regions is decreasing in $\beta$ as $k$ increases. Similarly, when we consider $e^{M P}$ and $e^{M I}$, we see that as $\alpha$ increases, the $e^{M P}<e^{M I}$ area increases with $\alpha$. 

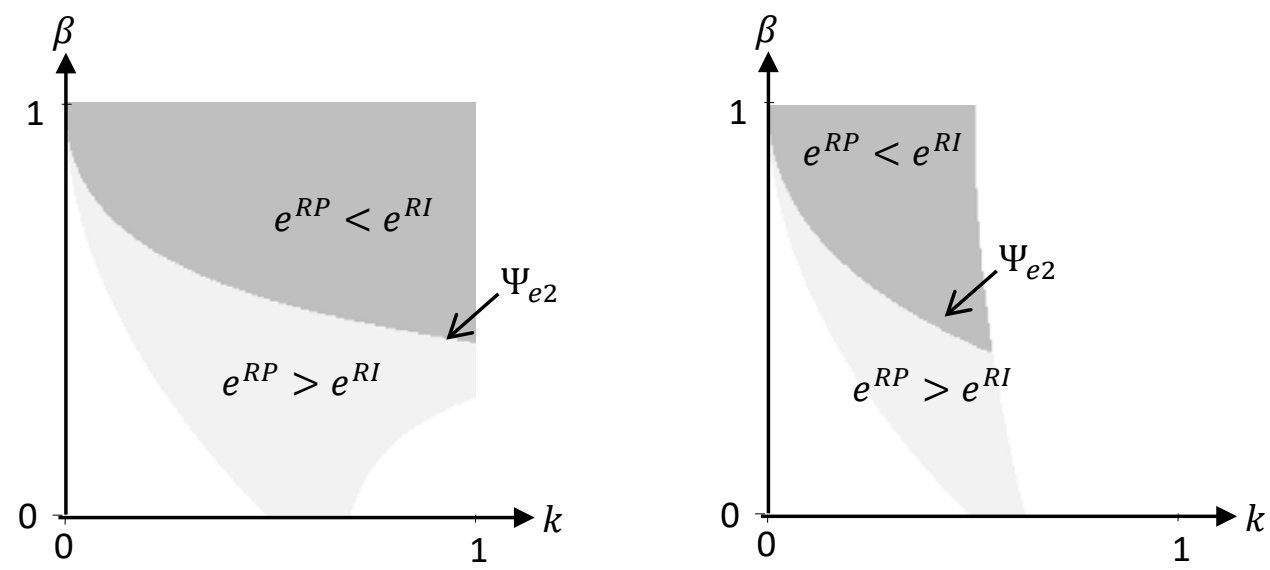

Figure 1: Comparison of $e^{R P}$ and $e^{R I}$ when $\alpha=0.5$ (left) and when $\alpha=1$ (right)
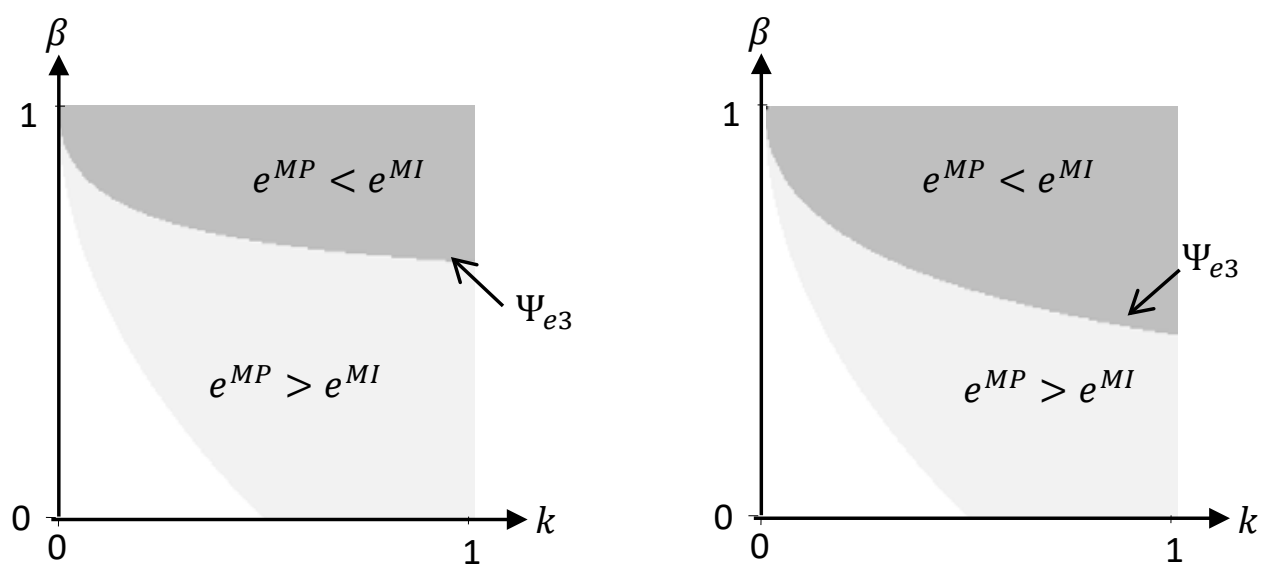

Figure 2: Comparison of $e^{M P}$ and $e^{M I}$ when $\alpha=0.5$ (left) and when $\alpha=1$ (right)

\subsection{Profit Comparisons}

In this subsection, we compare the profits of the firms and the government under different systems. Specifically, Proposition 2 examines the impact of different types of subsidy (comparisons among systems $x P, x I$, and $x B$ ), and then Proposition 3 studies the differences between retailer-initiated and manufacturer-initiated scenarios (comparisons between systems $R y$ and $M y)$. Recall that $x \in\{R, M\}$ represents whether the Retailer or the Manufacturer initiates the 
collaboration, and $y \in\{P, I, B\}$ denotes whether the government provides a Per-unit Production subsidy, an Innovation effort subsidy or Both types of subsidy.

\section{Proposition 2:}

The profit of the manufacturer is greatest under the per-unit subsidy, followed by when both subsidies are present, and least when only innovation effort subsidy is present, regardless of how the RJV is formed. Formally we write:

a. $\pi_{M}^{x P}>\pi_{M}^{x B}>\pi_{M}^{x I}$.

The similar result holds for the retailer when it initiates the RJV, and retailer's profits are all equal when the manufacturer initiates the RJV. Formally we write:

b. $\pi_{R}^{R P}>\pi_{R}^{R B}>\pi_{R}^{R I} ; \pi_{R}^{M P}=\pi_{R}^{M I}=\pi_{R}^{M B}$.

The government profit is greatest when both subsidies are present, and only in certain cases, $\Psi_{x 2}>0$, may we determine if the government prefers per-unit subsidy over innovation effort subsidy. Formally we write:

c. $\pi_{G}^{x B}>\max \left[\pi_{G}^{x P}, \pi_{G}^{x I}\right]$ and $\pi_{G}^{x P}>\pi_{G}^{x I} \Leftrightarrow \Psi_{\mathrm{x} 2}>0$.

Where $\Psi_{M 2}$ and $\Psi_{R 2}$ is defined in the proof.

First, regardless of which firm initiate the innovation project, Proposition 2a shows that the manufacturer is always better off when the government provides a per-unit subsidy. On the other hand, the manufacturer is worse off when the government provides a subsidy for innovation. When the government provides both types of subsidy, then the manufacturer's profit is between the two cases where the government only provides a single type of subsidy. This is because when the retailer initiates the RJV, the manufacturer would have the largest subsidy level when the government provides per-unit tax credit for each produced unit, and would have 
the smallest subsidy fraction when the government encourages innovation by subsidizing the cost of innovation (cf. Proposition 1b). With a larger subsidy level, the manufacturer thus has more profit under case RP than under case RI. On the other hand, when the manufacturer initiates the RJV, the effect on the manufacturer's profit from the innovation cost dominates the effect from subsidy. Specifically, the manufacturer would share the smallest amount of innovation cost when the government provides per-unit subsidy, but it is responsible for the largest share of innovation cost when the government subsidizes innovation (cf. Proposition 1c). As a result, the manufacturer has more profit under case MP than under case MI because of this impact from the innovation cost.

Even though the follower covers the smallest fraction of the cost when the government uses innovation subsidy as compared to when the government uses other forms of subsidy (cf. Proposition 1), both firms are always (weakly) worse off under system $x I$ regardless of which firm initiates the collaboration. Proposition $2 \mathrm{~b}$ shows that the retailer is indifferent between government's subsidy policies whenever the manufacturer initiates sustainability innovation. This is because the manufacturer would set the collaboration level $\lambda$ without violating the retailer's participation constraint $\left(\pi_{R} \geq 0\right)$, and we show in the derivation of the equilibrium solution (cf. Appendix A) that the retailer's participation constraint is always binding. As a result, the retailer always has zero profit whenever the manufacturer initiates sustainability innovation, so the retailer is indifferent to the government's subsidy policies.

When the government uses a single type of subsidy (system $x P$ or $x I$ ), it is better off using the per-unit tax subsidy when $\Psi_{\mathrm{R} 2}>0$, if the retailer initiates collaboration, or when $\Psi_{M 2}>0$, if the manufacturer initiates collaboration. Note that $\Psi_{\mathrm{R} 2}$ is obtained from comparing between $\pi_{G}^{R P}$ and $\pi_{G}^{R I}$; similarly, $\Psi_{\mathrm{M} 2}$ is obtained from comparing between $\pi_{G}^{M P}$ and $\pi_{G}^{M I}$. These 
two thresholds are a function of all model parameter values: the base innovation effort cost $(k)$, the marginal benefit in effort level to government's objective function $(\alpha)$ and the impact of effort to the per-unit production cost $(\beta)$. Moreover, the solution to $\Psi_{\mathrm{R} 2}=0$ and the solution to $\Psi_{\mathrm{M} 2}=0$ are a function of $k$ is a non-monotonic function in $\beta$, such that it decreases in $k$ when innovation is cheap, and increases in $k$ when innovation is costly.

We capture this tradeoff in Figure 3, using a coloring scheme analogous to Figures 1 and 2. As in the left diagrams of Figures 3 and 4 (government benefit from innovation, $\alpha$, is small), the condition $\Psi_{\mathrm{x} 2}>0, x \in\{M, R\}$ is satisfied when per-unit production cost, $\beta$, is large and the base innovation cost, $k$, is small. However, when the government can benefit more from innovation ( $\alpha$ is large), the impact of base innovation cost, $k$, diminishes (the marginal change in $\Psi_{x 2}$ with respect to $k$ decreases, i.e., the line $\Psi_{x 2}=0$ above which $\pi_{G}^{x P}<\pi_{G}^{x I}, x \in\{R, M\}$, below which $\pi_{G}^{x P}>\pi_{G}^{x I}$ ). Furthermore, regardless of the impact of innovation on production cost, recall from Propositions 2b,c that using a single type of subsidy always results in a more extreme collaboration level, in which the RJV follower is responsible for a larger (smaller) fraction of the cost/subsidy when the government uses per-unit production (innovation effort) subsidy relative to when both types of subsidy are present. The government benefits most from using both types of subsidy (system $x B$ ) as it has more flexibility in manipulating the collaboration level by carefully selecting both forms of subsidy, $t$ and $\Delta k$. Therefore, the government should use both types of subsidy whenever possible, regardless of the collaboration dynamics (cf. Proposition 2c). However, both firms are (weakly) worse off, so using both types of subsidy is never Pareto optimal to all parties. 

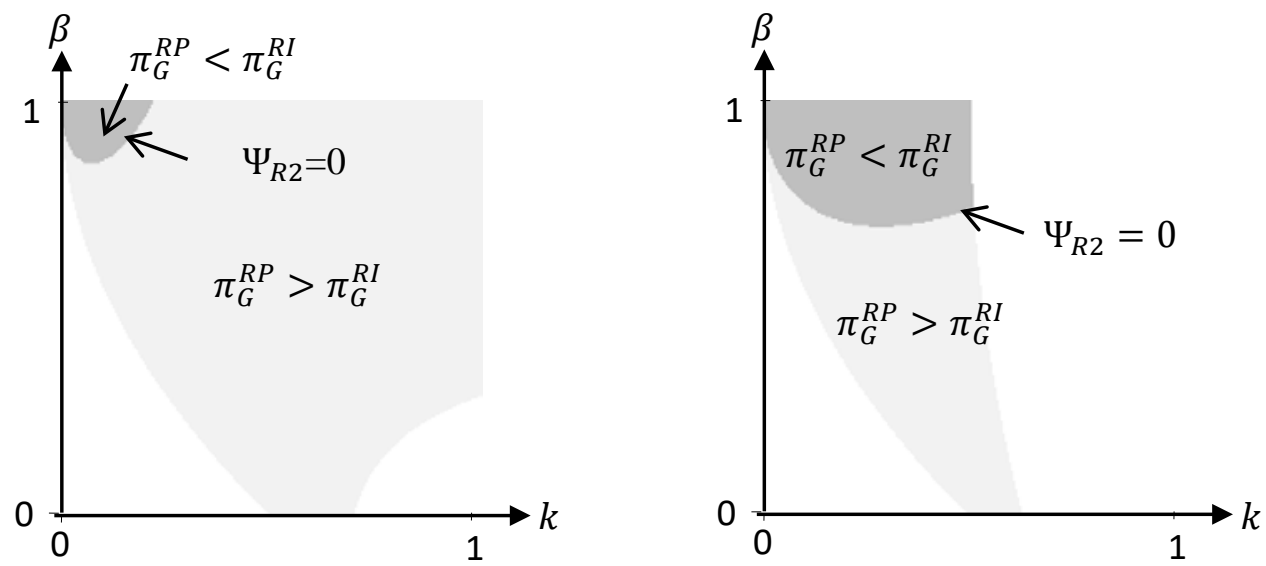

Figure 3: Comparison of $\pi_{G}^{R P}$ and $\pi_{G}^{R I}$ when $\alpha=0.5$ (left) and when $\alpha=1$ (right)
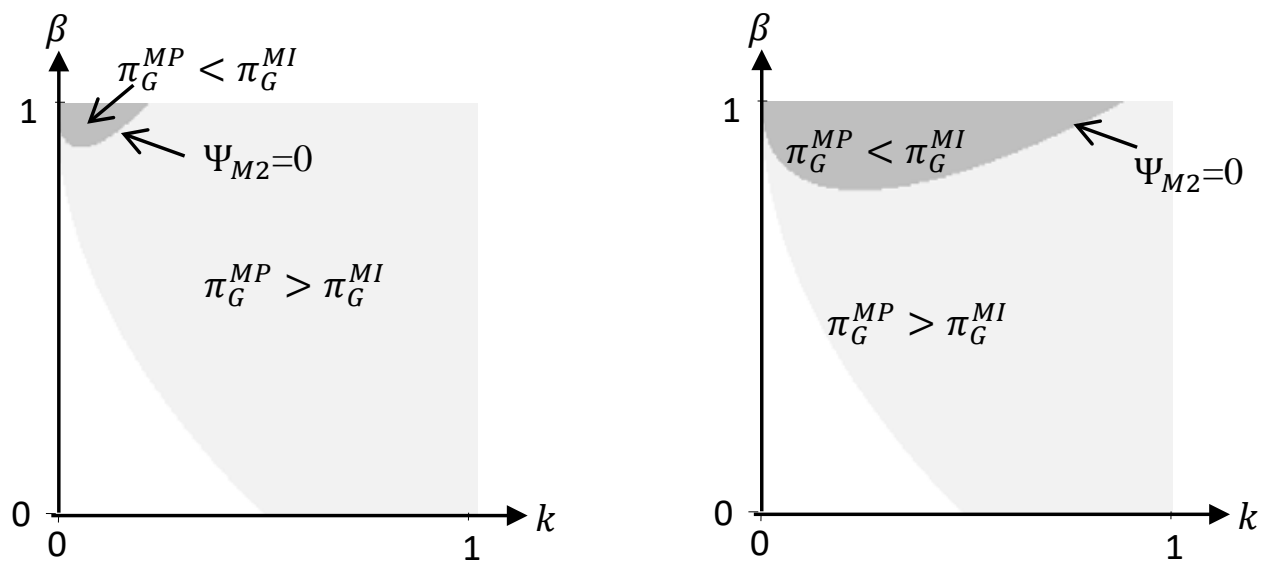

Figure 4: Comparison of $\pi_{G}^{M P}$ and $\pi_{G}^{M I}$ when $\alpha=0.5$ (left) and when $\alpha=1$ (right)

\section{Proposition 3:}

The profit of the manufacturer is greatest when it initiates the RJV, relative to when the retailer initiates the RJV except for the case when per-unit subsidy is present along with a mild feasibility condition, $\Psi_{2}<0$. Formally we write:

a. $\pi_{M}^{R P}>\pi_{M}^{M P} \Leftrightarrow \Psi_{2}<0 ; \pi_{M}^{M I}>\pi_{M}^{R I} ; \pi_{M}^{M B}>\pi_{M}^{R B}$.

The retailer is always better off initiating the RJV, when compared to the manufacturer initiating the RJV. Formally we write: 
b. $\pi_{R}^{R y}>\pi_{R}^{M y}$.

The government prefers to have a retailer initiated $R J V$ when only per-unit subsidy is present and the innovation cost is sufficiently small, $k<\Psi_{3}$, or the marginal benefit of innovation effort and increase in per-unit product cost are sufficiently small, $4 \alpha+\beta<1$. The government is indifferent to the RJV formation dynamics when either only innovation effort subsidy or both subsidies is present. Formally, we write :

c. $\pi_{G}^{R P}>\pi_{G}^{M P} \Leftrightarrow 4 \alpha+\beta<1$ OR $k<\Psi_{3} ; \pi_{G}^{R I}=\pi_{G}^{M I} ; \pi_{G}^{R B}=\pi_{G}^{M B}$.

Where $\Psi_{2}$ and $\Psi_{3}$ are defined in the proof.

One might expect that a firm is always better off being the RJV initiator because it has a first-mover advantage in deciding the fraction of cost/subsidy for which it is responsible. This is exactly the case for the retailer as depicted in Proposition 3b since when the manufacturer is the first-mover, it controls both effort and share levels thus leaves zero profit to the retailer, i.e., $\pi_{R}^{M y}=0$. Yet Proposition 3a demonstrates that such intuition may not hold in some cases. In particular, when the government uses per-unit tax subsidy alone (system $x P$ ), the manufacturer may be worse off being the RJV initiator (first mover) if $\Psi_{2}<0$, depicted in the light grey area in Figure 5. Note that the threshold $\Psi_{2}$ is simply a multiplier of the difference between the manufacturer's profit when the retailer initiates RJV (case RP) and when the manufacturer initiates RJV (case MP) for the situation where the government provides per-unit tax credit. Given that the profit functions $\pi_{M}^{R P}$ and $\pi_{M}^{M P}$ are functions of the innovation effort cost $(k)$, the marginal benefit in effort level $(\alpha)$ and the impact of effort to production cost $(\beta)$, the threshold $\Psi_{2}$ is also a function of these three parameters. The negativity condition, $\Psi_{2}<0$, is likely to hold when the base innovation cost, $k$, or the post-innovation per-unit production cost change, $\beta$, is sufficiently small. This follows from the fact that the per-unit tax credit subsidy is much larger 
when the retailer initiates $\left(t^{R P} \gg t^{M P}\right)$, leading to a larger sustainability innovation effort, so the demand is larger when the retailer initiates $\left(D^{R P}>D^{M P}\right)$. Further, the manufacturer receives a larger fraction of the per-unit tax credit, despite covering a larger fraction of the innovation cost $\left(\lambda^{R P}>\lambda^{M P}\right)$. As a result, the manufacturer is better off being the follower in the supply chain relationship.
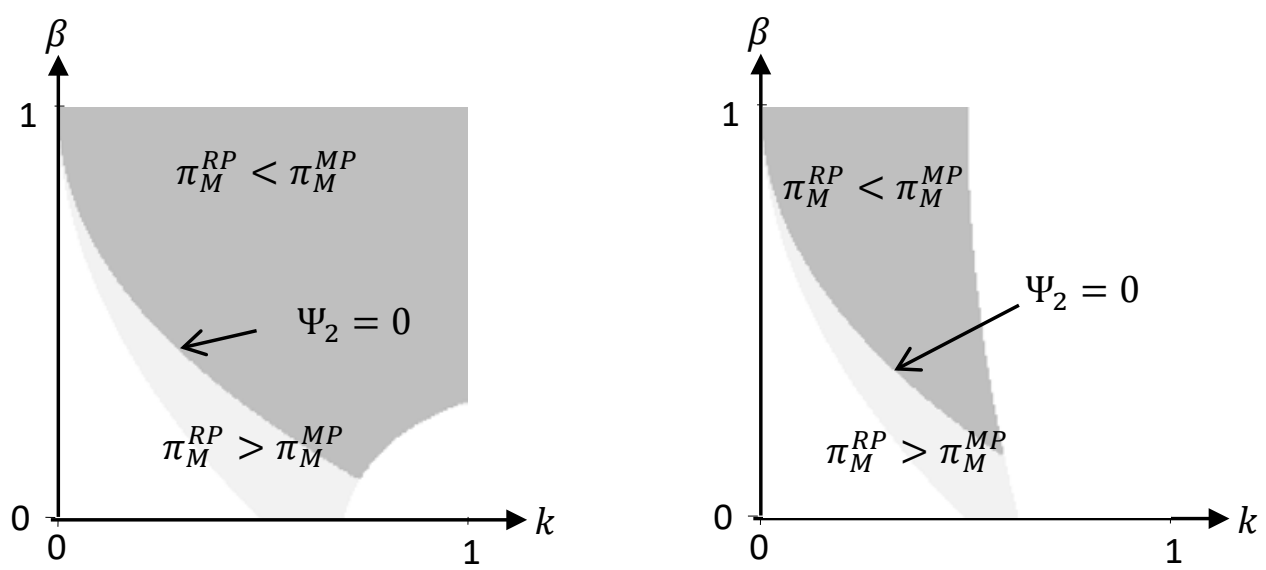

Figure 5: Comparison of $\pi_{M}^{R P}$ and $\pi_{M}^{M P}$ when $\alpha=0.5$ (left) and when $\alpha=1$ (right)

Note that the government is also better off with a retailer-initiated RJV when the base innovation cost, $k$, or the post-innovation per-unit production cost change, $\beta$, is sufficiently small ( $k<\Psi_{3}$ ), as presented in Figure 6. We note that the threshold $\Psi_{3}$ is a function of $\alpha$ and $\beta$, and it decreases in the impact of effort to the per-unit production cost $(\beta)$. As a result, the government is more likely better off if the manufacturer initiates collaboration when the government uses per-unit subsidy and when sustainability innovation is costly ( $k$ large). Moreover, the ranges of $k$ and $\beta$ that result in the government preferring a retailer-initiated RJV are reduced as the government's benefit from innovation, $\alpha$, increases. 

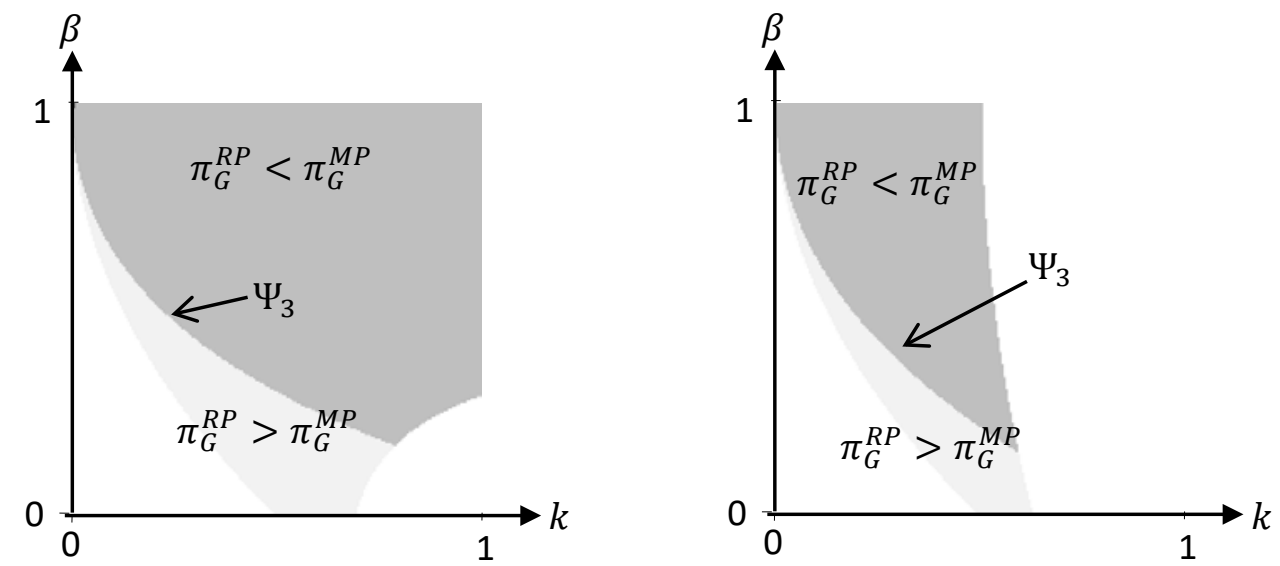

Figure 6: Comparison of $\pi_{G}^{R P}$ and $\pi_{G}^{M P}$ when $\alpha=0.5$ (left) and when $\alpha=1$ (right)

Even though the innovation subsidy, $\Delta k$, depends on which firm initiates the RJV collaboration (cf. Proposition B1b), the government is agnostic about the collaboration dynamics when it provides innovation subsidy (systems $x I$ and $x B$ ). This is because the optimal levels of innovation effort and price are independent of which firm initiates the collaboration, so demand (and hence, customer surplus) is the same $\left(D^{R I}=D^{M I}\right.$ and $\left.D^{R B}=D^{M B}\right)$. Moreover, the profit of the entire supply chain is similar, differing only by $\left(\Delta k^{R B}-\Delta k^{M B}\right)\left(e^{x B}\right)^{2}$ between the two collaboration dynamics despite the fact that each individual firm's profit depends on which firm initiates collaboration. As a result, the government is indifferent to the collaboration dynamics when it provides innovation subsidy.

We note that the game sequence of our main model does not include the kind of subsidy the government should provide. However, given the ranking of the government's objective function in Propositions 2 and 3, it suggests that whenever the R\&D effort increases per-unit production cost, the government should provide both types of subsidy. 


\section{Managerial Insights and Future Research Directions}

In this paper, we present a sustainable supply chain model that considers government subsidy, RJV formation dynamics, innovation effort, and pricing decisions. To our knowledge, this is the first work in the OM literature to consider all four decisions in one model. The theoretical contribution of our paper is as follows. Our paper extends the literature of sustainability that studies social welfare and regulation by considering subsidy for both upfront innovation cost and post-innovation per-unit production cost. Moreover, our paper differs from the stream of literature that examines the area of collaboration innovation by investigating how government policy influences the collaborative innovation between these two supply chain entities. To the best of our knowledge, this is the first model that jointly considers (1) innovation and production subsidies, and (2) two types of supply chain collaboration (manufacturer initiates innovation and retailer initiates innovation). This helps to address two of the four research gaps, the largely ignored market forces and vertically aligned sustainable strategies, identified by Tang and Zhou (2012). The following section summarizes the insights that managers may glean from this study, as well as our recommendations for future research.

\subsection{Managerial Insights}

As discussed in the introduction, our work is motivated by governments' push for sustainability via their industries and policies and executives desire to collaborate in sustainable innovation (Kiron et al., 2015; Rossini et al., 2016). The first key message of our paper is that the government should never use both types of subsidies simultaneously for any cost-reduction R\&D effort. We are beginning to see this in the European Union's Common Agricultural Policy (CAP) reform as it shifts from both product-based and producer-based subsidies to producer 
support/subsidies (European Commission, 2013) (i.e., from something akin to having both subsidies present to only innovation subsidies in our paper). Another insight that government policy makers may take away from our results (and from Proposition 3 in particular) is that regardless of how an industrial sector decides to develop sustainable products or how RJVs are formed, so long as cost-increasing R\&D effort is present, the government is indifferent to how the RJV is formed as long as innovation effort subsidy is present. We see that certain governments have already embraced this approach, as in the American Recovery and Reinvestment Act (US Congress \& U.S. Congress, 2009) and Innovate UK, which provide subsidy for sustainability efforts. On the other hand, when the government only uses per-unit subsidy, then a retailer-initiated RJV is preferable when innovation is not too costly. We realize that most government incentive policies provide multipronged subsidy; however, for smaller, potentially budget-constrained governments, ensuring that effort subsidy is present is one key takeaway from our work.

Another group that may benefit from our work is company leaders looking to respond to government incentive programs by undertaking research in RJVs. For instance, when Walmart collaborated with its suppliers (such as Clorox) to create smaller bleach bottles, it initiated RJVs as part of a sustainability program. However, when government subsidy is present, it is unclear which party should initiate the venture. A myopic view of this issue would be to assume that in all circumstances, the initiating party has first-mover advantage. As noted in Proposition 3, a manufacturer should not initiate the RJV when only per-unit government subsidy is present, the innovation cost is sufficiently small, and the post-innovation increase in per-unit cost is small. Even though the manufacturer is better off not initiating the venture, it covers more of the RJV cost by not initiating the RJV than by initiating it (Proposition 1). 
Finally, many industries have lobbyists to help present their views to government organizations; for example, Walmart has various firms lobbying on its behalf (Center for Responsive Politics, 2015). These lobbyists may request that governments provide subsidy in order to help firms satisfy various government policies or initiatives. One insight that lobbyists may glean from our analysis is that the supply chain partners are better off when only per-unit government subsidy is present (Proposition 2). However, if effort subsidy is present, the supply chain partners are better off having per-unit subsidy present as well, as governments are better off using both forms of subsidy.

These are compelling insights not only for policy makers, but also for industry leaders. This is a much needed first step in sustainable supply chain research, and there are numerous possible directions for future study.

\subsection{Future Research Directions}

The first natural extension to the model presented here is to consider different bargaining strengths among the supply chain partners. In this paper, we assume that the initiating partner (either manufacturer or retailer) holds all of the bargaining power in the relationship. We have performed preliminary analysis considering the case where both firms have equal bargaining power. Based on the optimal solution under the bargaining game, we examine the impact on our results when the manufacturer and the retailer have the same bargaining power. Our objective is to compare different government subsidies under the bargaining game (The results are available from the authors upon request). Our extension shows that when two firms have equal bargaining power, the innovation-only subsidy leads to the lowest collaboration level in retailer-initiated (cf. Proposition 1b) and the highest collaboration level in manufacturer-initiated (cf. Proposition 1c). 
Moreover, we find that the sustainability effort is higher when providing both forms of subsidies than simply providing innovation subsidy. The insight from the comparison of the effort level for per-unit subsidy and for innovation subsidy continues to hold when the two firms have equal power. The rankings of the manufacturer's profit and the retailer's profit are the same as the retailer-initiated and the manufacturer-initiated scenarios. In terms of the ranking of the government's profit. Similar to our findings in Proposition 2c, we find that the government is always better off when providing both types of subsidies than providing innovation subsidy. Lastly, the comparison between government's profit when providing a single type of subsidy is not affected when the two firms have equal bargaining power. With that being said, it would be interesting to consider a complete analysis about how the firms and government are affected as a function of various possible bargaining power. A second extension of this model is to consider a temporal component. As discussed in the introduction, there are multiple forms of government subsidy available. Two forms of subsidy are tax subsidy (given directly to the innovating firm(s)) and tax (usually import) on competing firms. In both cases, the government is trading future gains for current gains, and incorporating these tradeoffs into a sustainable supply chain model could be of interest. Lastly, in the subsidy considered here, we assume no competition from other governments as well as no competition to the supply chain partners. In actuality, competition exists not only from other governments but also from other supply chain partners; for example, GM and its dealership network and Ford and its dealership network directly compete with each other. Incorporating competition of any form into the presented model will also yield further insights. 


\section{Appendix A: Derivation of Equilibrium}

We start by analyzing two general cases: the retailer-initiated collaboration with both types of subsidy $(R B)$, and the manufacturer-initiated collaboration with both types of subsidy $(M B)$. For the ease of analysis and presentation, we sometimes solve same player's simultaneous decisions sequentially, e.g., solving w first then e (two joint decisions) in step 3 of the retailer initiated case. We do this to take advantage of some structural properties that facilitate the analysis but note that the final solutions are the same regardless of the order of how they are solved as long as it is the same decision maker. We then provide brief analysis and the equilibrium solutions of the other four cases ( $R P, R I, M P$, and $M I)$, following the same backward induction solution procedure. To focus on the non-trivial region where efforts are positive, for this paper, we only consider the region where $\beta<1$. Otherwise, the optimal solution shows that the per-unit increase in production cost due to sustainability innovation is too high and the firms would never innovate.

\section{Retailer-Initiated Collaboration with Both Types of Subsidy (RB)}

Pricing decisions ( $p$ and $w$ ): First, in Step 4 of the game sequence, since $\pi_{R}$ is concave in $p$, solving the first-order optimality condition (FOC) for $p, \frac{\partial \pi_{R}}{\partial p}=0$, yields the optimal retail price, $p^{*}(w, e, \lambda, t, \Delta k)$. (Note that for ease of presentation, we suppress some or all of the arguments unless confusion may arise.) In Step $3, \pi_{M}$ is concave in $w$ and solving the FOC $\frac{\partial \pi_{M}}{\partial w}=0$ gives:

$$
w^{*}(e, \lambda)=\frac{1+(1+\beta) e+t(1-2 \lambda)}{2} \text { and } p^{*}(e)=\frac{3+(3+\beta) e-t}{4} .
$$

The wholesale price, $w$, is increasing as either the production cost, $\beta$, or effort, $e$, increase, and is decreasing as the Manufacturer's RJV share, $\lambda$, increases. The effect of per-unit production subsidy, $t$, on $w$ depends on $\lambda$. When the manufacturer receives more (or less) than half of the unit subsidy, it reduces (or increases) its price charged to the retailer. We continue 
with the process to find $e^{*}(\lambda)$. Substituting $p^{*}(e)$ and $w^{*}(e, \lambda)$ into $\pi_{M}$ and solving for $e$ in $\frac{\partial \pi_{M}}{\partial e}=0$ gives the optimal effort level, $e^{*}(\lambda)=\frac{(1+t)(1-\beta)}{8 \lambda(\mathrm{k}-\Delta k)-(1-\beta)^{2}}$.

Innovation $R J V$ decisions $(\lambda)$ : In Step 2 the retailer decides the collaboration level, $\lambda$. Substituting $e^{*}(\lambda)$ into $\pi_{R}$ and solving for the FOC $\frac{\partial \pi_{R}}{\partial \lambda}=0$ gives:

$$
\lambda^{*}(\Delta k)=1-\frac{(1-\beta)^{2}}{16(k-\Delta k)} .
$$

Note that the share level, $\lambda$, only depends on innovation subsidy, $\Delta k$, but not on production subsidy, $t$. In particular, the retailer will increase its share $(1-\lambda)$ for smaller production cost increases or lower net innovation cost $(k-\Delta k)$. Given the equilibrium collaboration level, we rewrite the manufacturer's effort level as: $e^{*}(t, \Delta k)=\frac{(1+t)(1-\beta)}{8(k-\Delta k)-\frac{3}{2}(1-\beta)^{2}}$. From the equilibrium effort decision, we note that effort level depends on both subsidies. Though collaboration does raise innovation effort level (for any given subsidy, from $\left.e^{*}\right|_{\lambda=1}=$ $\frac{(1+t)(1-\beta)}{8(k-\Delta k)-(1-\beta)^{2}}$ with no collaboration to $\left.e^{*}\right|_{\lambda=\lambda^{*}}=\frac{(1+t)(1-\beta)}{8(k-\Delta k)-\frac{3}{2}(1-\beta)^{2}}$ with collaboration), collaboration helps more for small production cost increases (small $\beta$ ) as the retailer strategically shares less as production cost increases.

Government subsidy decisions ( $t$ and $\Delta k$ ): Lastly, in Step 1, substituting all of the above optimal decision variables into $\pi_{G}$ and solving the two FOCs $\left(\frac{\partial \pi_{G}}{\partial t}=0\right.$ and $\left.\frac{\partial \pi_{G}}{\partial \Delta k}=0\right)$ simultaneously for $t$ and $\Delta k$ give the optimal solutions:

$$
\begin{gathered}
t^{R B}=\frac{3[2 k+\alpha(1-\beta)]}{2 k-(1-\beta)^{2}}, \Delta k^{R B}=\frac{16 k \alpha-(9 \alpha+1-\beta)(1-\beta)^{2}}{16(\alpha+1-\beta)}, \\
\lambda^{R B}=\frac{8[2 k+\alpha(1-\beta)]}{16 k+(9 \alpha+1-\beta)(1-\beta)}, e^{R B}=\frac{\alpha+1-\beta}{2 k-(1-\beta)^{2}},
\end{gathered}
$$




$$
\begin{aligned}
& \pi_{M}^{R B}=\frac{[2 k+\alpha(1-\beta)][8 k+(1-\beta)(3 \alpha-(1-\beta))]}{2\left[2 k-(1-\beta)^{2}\right]^{2}} \pi_{R}^{R B}=\frac{[8 k+(5 \alpha+(1-\beta))(1-\beta)][8 k+(1-\beta)(3 \alpha-(1-\beta))]}{16\left[2 k-(1-\beta)^{2}\right]^{2}}, \text { and } \\
& \pi_{G}^{R B}=\frac{2 k+\alpha[\alpha+2(1-\beta)]}{2\left[2 k-(1-\beta)^{2}\right]} .
\end{aligned}
$$

In equilibrium, together positive retail price $p^{R B}=\frac{\beta(\alpha+1-\beta)}{2 k-(1-\beta)^{2}}$ and effort $e^{R B}=\frac{\alpha+1-\beta}{2 k-(1-\beta)^{2}}$

require $\beta>0$ and $k>\frac{(1-\beta)^{2}}{2}$. In addition, second-order optimality condition requires $\beta<1$ to ensure that we are finding global maxima when deriving equilibrium decisions. We note that boundary solutions in the general case reduce to special cases that are analyzed separately (in the appendix as the solving procedures are similar for RI and RP), e.g., RP is RB with $\Delta k^{R B}=0$ and $\mathrm{RI}$ is $\mathrm{RB}$ with $t^{R B}=0$. This implies that for cost-reducing (negative) $\beta$ and thus a negative retail price, the government would never use both subsidies simultaneously. For ease of exposition in the main text, we present and discuss only interior solutions that satisfy joint technical conditions for all cases, in particular, $0<\beta<1$. However, using the conditions on the non-negativity of other decision variables (e.g., $\Delta k, e, p, \lambda$ ) one may show that the range of $\beta$ range may be relaxed under special cases. When all technical conditions are met, all three parties’ profits are increasing in $\alpha$, suggesting that sustainability innovation can be a win-win strategy for both the government (society) and supply chain partners, if the government (society) benefits significantly from sustainable business practices.

\section{Manufacturer-Initiated Collaboration with Both Types of Subsidy (MB)}

Pricing decisions ( $p$ and $w$ ): Same as in the $R B$ case.

Innovation effort and RJV decisions (e and $\lambda$ ): In Step 2, with $p=p^{*}(e)$ and $w=w^{*}(e, \lambda), \pi_{M}$ is decreasing in $\lambda$ while $\pi_{R}$ is increasing in $\lambda$ for any given innovation effort level, $e$. This means 
that the manufacturer will reduce $\lambda$ until $\pi_{R}=0$, the retailer's outside option, to satisfy individual rationality. At which point, solving $\left.\frac{\partial \pi_{M}}{\partial e}\right|_{\lambda: \pi_{R}=0}=0$ yields:

$$
\lambda^{*}(\Delta k)=1-\frac{16(k-\Delta k)}{9(1-\beta)^{2}} \text { and } e^{*}(t, \Delta k)=\frac{3(1+t)(1-\beta)}{16(k-\Delta k)-3(1-\beta)^{2}} .
$$

Note that $\lambda^{*}(\Delta k)$ may be negative, meaning that on the one hand, the manufacturer would receive money from the retailer, and the amount of money received is a function of the innovation level. This money transaction may occur during the product development phase, and so it can be interpreted as the scenario where the retailer provides a short-term loan to the manufacturer to cover the innovation cost. On the other hand, the manufacturer also needs to pay money to the retailer, and this amount is a function of the government's subsidy. One realistic scenario is that this transaction happens after demand is realized and the manufacturer pays back the short term loan that it receives from the retailer during the product development phase.

Government subsidy decisions ( $t$ and $\Delta k$ ): Lastly, in Step 1, as in the $R B$ case, solving the two FOCs, $\frac{\partial \pi_{G}}{\partial t}=0$ and $\frac{\partial \pi_{G}}{\partial \Delta k}=0$, simultaneously gives the optimal solutions:

$$
\begin{gathered}
t^{M B}=\frac{3[2 k+\alpha(1-\beta)]}{2 k-(1-\beta)^{2}}, \Delta k^{M B}=\frac{2 k[2 \alpha-(1-\beta)]-3 \alpha(1-\beta)^{2}}{4(\alpha+1-\beta)}, \\
\lambda^{M B}=\frac{-8 k-(1-\beta)[\alpha-3(1-\beta)]}{3(1-\beta)(\alpha+1-\beta)}, e^{M B}=\frac{\alpha+1-\beta}{2 k-(1-\beta)^{2}}, \\
\pi_{M}^{M B}=\frac{3[2 k+\alpha(1-\beta)][8 k+(1-\beta)(3 \alpha-(1-\beta))]}{4\left[2 k-(1-\beta)^{2}\right]^{2}}, \pi_{R}^{M B}=0, \text { and } \pi_{G}^{M B}=\frac{2 k+\alpha[\alpha+2(1-\beta)]}{2\left[2 k-(1-\beta)^{2}\right]} .
\end{gathered}
$$

Since the equilibrium retail price is the same and technical conditions are similar as in the RB case, we omit their interpretations for this case but note that we compare and discuss results across all six cases next. 
System $\boldsymbol{R P}$ : For notation convenience, define $\Gamma_{R P}=256 k^{2}-160 k(1-\beta)^{2}+17(1-\beta)^{4}$. First, note that $\pi_{R}$ is concave in $p$, so we solve $\frac{\partial \pi_{R}}{\partial p}=0$ for the optimal $p$. Second, $\pi_{M}$ is concave in $w$, and we solve $\frac{\partial \pi_{M}}{\partial w}=0$ for the optimal $w$. Solving for the FOC $\frac{\partial \pi_{M}}{\partial e}=0$ gives $e=$ $\frac{(1+t)(1-\beta)}{8 k \lambda-(1-\beta)^{2}}$. Similarly, solving the FOC $\frac{\partial \pi_{R}}{\partial \lambda}=0$ gives $\lambda=1-\frac{(1-\beta)^{2}}{16 k}$. Lastly, solving for the FOC $\quad \frac{\partial \pi_{G}}{\partial t}=0 \quad$ gives $\quad t=\frac{768 k^{2}-(5+96 \alpha-5 \beta)(1-\beta)^{3}+32 k(1-\beta)(16 \alpha+3 \beta-3)}{\Gamma_{R P}} \quad, \quad e=$ $\frac{8(1-\beta)(16 k+(8 \alpha+\beta-1)(1-\beta))}{\Gamma_{R P}}, \lambda=1-\frac{(1-\beta)^{2}}{16 k}, \pi_{M}=\frac{2\left(16 k-(1-\beta)^{2}\right)(16 k+(8 \alpha+\beta-1)(1-\beta))^{2}\left(16 k-3(1-\beta)^{2}\right)}{\Gamma_{R P}^{2}}$, $\pi_{R}=\frac{\left(16 k-3(1-\beta)^{2}\right)(16 k+(8 \alpha+\beta-1)(1-\beta))^{2}\left(16 k+(1-\beta)^{2}\right)}{\Gamma_{R P}^{2}}$, and $\pi_{G}=\frac{(16 k+(8 \alpha+\beta-1)(1-\beta))^{2}}{2 \Gamma_{R P}}$. Note that at the optimal solution, $\frac{\partial^{2} \pi_{M}}{\partial e^{2}}=-\frac{16 k-3(1-\beta)^{2}}{8}, \frac{\partial^{2} \pi_{R}}{\partial \lambda^{2}}=\frac{-2048 k^{2}(1-\beta)^{2}(16 k+(8 \alpha+\beta-1)(1-\beta))^{2}}{\left(16 k-3(1-\beta)^{2}\right) \Gamma_{R P}^{2}}$, and $\frac{\partial^{2} \pi_{G}}{\partial t^{2}}=\frac{-\Gamma_{R P}}{16\left(16 k-3(1-\beta)^{2}\right)^{2}}$. It can be shown that $16 k-3(1-\beta)^{2}>0 \Leftrightarrow k>\frac{3(1-\beta)^{2}}{16}, \Gamma_{R P}>0 \Leftrightarrow$ $k>\frac{5+2 \sqrt{2}}{16}(1-\beta)^{2}$ or $k<\frac{5-2 \sqrt{2}}{16}(1-\beta)^{2}$, and $\frac{5+2 \sqrt{2}}{16}(1-\beta)^{2}>\frac{3(1-\beta)^{2}}{16}>\frac{5-2 \sqrt{2}}{16}(1-\beta)^{2}$, so all SOCs are satisfied if and only if $k>\frac{5+2 \sqrt{2}}{16}(1-\beta)^{2}$.

System RI: For notation convenience, define $\Gamma_{R I}=32 k-7(1-\beta)^{2}$. First, note that $\pi_{R}$ is concave in $p$, so we solve $\frac{\partial \pi_{R}}{\partial p}=0$ for the optimal $p$. Second, $\pi_{M}$ is concave in $w$, and we solve $\frac{\partial \pi_{M}}{\partial w}=0$ for the optimal $w$. Solving for the FOC $\frac{\partial \pi_{M}}{\partial e}=0$ gives $e=\frac{(1-\beta)}{8(k-\Delta k) \lambda-(1-\beta)^{2}}$. Similarly, solving the FOC $\frac{\partial \pi_{R}}{\partial \lambda}=0$ gives $\lambda=1-\frac{(1-\beta)^{2}}{16(k-\Delta k)}$. Lastly, solving for the FOC $\frac{\partial \pi_{G}}{\partial t}=0$ gives $\Delta k=\frac{16 k(16 \alpha+3-3 \beta)-(7+48 \alpha-7 \beta)(1-\beta)^{2}}{16(7+16 \alpha-7 \beta)}, \quad e=\frac{16 \alpha+7(1-\beta)}{\Gamma_{R I}}, \lambda=\frac{32(2 k+\alpha-\alpha \beta)}{64 k+(7+48 \alpha-7 \beta)(1-\beta)}, \quad \pi_{M}=$ $\frac{2(2 k+\alpha-\alpha \beta)}{\Gamma_{R I}}, \pi_{R}=\frac{32 k+(7+32 \alpha-7 \beta)(1-\beta)}{16 \Gamma_{R I}}$, and $\pi_{G}=\frac{7 k+7 \alpha+8 \alpha^{2}-7 \alpha \beta}{\Gamma_{R I}}$. Note that at the optimal solution, $\frac{\partial^{2} \pi_{M}}{\partial e^{2}}=\frac{-(1-\beta) \Gamma_{12}}{4(16 \alpha+7(1-\beta))}, \frac{\partial^{2} \pi_{R}}{\partial \lambda^{2}}=\frac{-(1-\beta)(16 \alpha+7(1-\beta))(64 k+(48 \alpha+7-7 \beta)(1-\beta))^{2}}{16 \Gamma_{R I}^{3}}$, and $\frac{\partial^{2} \pi_{G}}{\partial \Delta k^{2}}=$ $\frac{-4(16 \alpha+7(1-\beta))^{4}}{(1-\beta)^{2} \Gamma_{R I}^{3}}$. It can be shown that $\Gamma_{R I}>0 \Leftrightarrow k>\frac{7(1-\beta)^{2}}{32}$, so all SOCs are satisfied if and only if $k>\frac{7(1-\beta)^{2}}{32}$.

System MP: For notation convenience, define $\Gamma_{M P}=8 k-3(1-\beta)^{2}$. First, note that $\pi_{R}$ is concave in $p$, so we solve $\frac{\partial \pi_{R}}{\partial p}=0$ for the optimal $p$. Second, $\pi_{M}$ is concave in $w$, and we solve 
$\frac{\partial \pi_{M}}{\partial w}=0$ for the optimal $w$. Note that $\frac{\partial^{2} \pi_{M}}{\partial e^{2}} \frac{\partial^{2} \pi_{M}}{\partial \lambda^{2}}-\left(\frac{\partial^{2} \pi_{M}}{\partial e \partial \lambda}\right)^{2}=-4 e^{2} k^{2}<0$, so this is a saddle point. Since $\pi_{M}$ decreases in $\lambda$ and $\pi_{R}$ increases in $\lambda$, M would set the smallest $\lambda$, such that $\pi_{R}=$ 0 . Then solving for the FOC $\frac{\partial \pi_{M}}{\partial e}=0$ gives $e=\frac{3(1+t)(1-\beta)}{16 k-3(1-\beta)^{2}}$. Lastly, solving for the FOC $\frac{\partial \pi_{G}}{\partial t}=$ 0 gives $t=\frac{3\left(16 k^{2}+2 k(1-\beta)(8 \alpha-1+\beta)-3 \alpha(1-\beta)^{3}\right)}{2 k \Gamma_{M P}}, e=\frac{3(1-\beta)(4 k+3 \alpha(1-\beta))}{2 k \Gamma_{M P}}, \lambda=1-\frac{16 k}{9(1-\beta)^{2}}, \pi_{M}=$ $\frac{3(4 k+3 \alpha-3 \alpha \beta)^{2}\left(16 k-3(1-\beta)^{2}\right)}{4 k \Gamma_{M P}^{2}}, \pi_{R}=0$, and $\pi_{G}=\frac{(4 k+3 \alpha-3 \alpha \beta)^{2}}{4 k \Gamma_{M P}}$. Note that at the optimal solution, $\frac{\partial^{2} \pi_{M}}{\partial e^{2}}=-\frac{16 k-3(1-\beta)^{2}}{8}$ and $\frac{\partial^{2} \pi_{G}}{\partial t^{2}}=\frac{-2 k \Gamma_{21}}{\left(3-16 k-6 \beta+3 \beta^{2}\right)^{2}}$. It can be shown that $16 k-3(1-\beta)^{2}>$ $0 \Leftrightarrow k>\frac{3(1-\beta)^{2}}{16}, \Gamma_{M P}>0 \Leftrightarrow k>\frac{3(1-\beta)^{2}}{8}$, and $\frac{3(1-\beta)^{2}}{8}>\frac{3(1-\beta)^{2}}{16}$, so all SOCs are satisfied if and only if $k>\frac{3(1-\beta)^{2}}{8}$.

System MI: For notation convenience, define $\Gamma_{M I}=32 k-7(1-\beta)^{2}$. First, note that $\pi_{R}$ is concave in $p$, so we solve $\frac{\partial \pi_{R}}{\partial p}=0$ for the optimal $p$. Second, $\pi_{M}$ is concave in $w$, and we solve $\frac{\partial \pi_{M}}{\partial w}=0$ for the optimal $w$. Note that $\frac{\partial^{2} \pi_{M}}{\partial e^{2}} \frac{\partial^{2} \pi_{M}}{\partial \lambda^{2}}-\left(\frac{\partial^{2} \pi_{M}}{\partial e \partial \lambda}\right)^{2}=-4 e^{2}(k-\Delta k)^{2}<0$, so this is a saddle point. Since $\pi_{M}$ decreases in $\lambda$ and $\pi_{R}$ increases in $\lambda$, M would set the smallest $\lambda$, such that $\pi_{R}=0$. Then solving for the FOC $\frac{\partial \pi_{M}}{\partial e}=0$ gives $e=\frac{3(1-\beta)}{16(k-\Delta k)-3(1-\beta)^{2}}$. Lastly, solving for the FOC $\frac{\partial \pi_{G}}{\partial \Delta k}=0$ gives $\Delta k=\frac{k(16 \alpha+1-\beta)-3 \alpha(1-\beta)^{2}}{16 \alpha+7-7 \beta}, e=\frac{16 \alpha+7-7 \beta}{\Gamma_{M I}}, \lambda=\frac{-32 k+(21+32 \alpha-21 \beta)(1-\beta)}{3(1-\beta)(16 \alpha+7-7 \beta)}$, $\pi_{M}=\frac{3(2 k+\alpha-\alpha \beta)}{\Gamma_{M I}}, \pi_{R}=0$, and $\pi_{G}=\frac{7 k+7 \alpha+8 \alpha^{2}-7 \alpha \beta}{\Gamma_{M I}}$. Note that at the optimal solution, $\frac{\partial^{2} \pi_{M}}{\partial e^{2}}=$ $\frac{-3(1-\beta) \Gamma 22}{8(16 \alpha+7-7 \beta)}$ and $\frac{\partial^{2} \pi_{G}}{\partial \Delta k^{2}}=\frac{-16(16 \alpha+7-7 \beta)^{4}}{9(1-\beta)^{2} \Gamma_{M I}^{3}}$. It can be shown that $\Gamma_{M I}>0 \Leftrightarrow k>\frac{7(1-\beta)^{2}}{32}$, so all SOCs are satisfied if and only if $k>\frac{7(1-\beta)^{2}}{32}$.

\section{Appendix B: Subsidy Parameters}

In this appendix, we examine the impact of different types of subsidy on a given supply chain structure (comparisons between systems $x P, x I$, and $x B$ ). Proposition B1 examines these impacts to the subsidy parameters, $t$ and $\Delta k$.

\section{Proposition B1:}


The optimal per-unit production subsidy by the government is always greatest when only perunit subsidy is offered, than when any other form of subsidy is offered. In addition, there exists a threshold, $\Psi_{1}$, on the effort cost, $k$, above which the per-unit government subsidy in the manufacturer initiated $R J V$ is higher than the retailer initiated $R J V$. Formally we write:

a. $t^{x P}>t^{R B}=t^{M B} ; t^{M P}>t^{R P} \Leftrightarrow k>\Psi_{1}$

Similarly, the innovation effort subsidy, $\Delta k$, is greatest when only it is present as opposed to when both subsidies are present. In addition, the innovation effort subsidy is greatest under the retailer initiated RJV dynamics, regardless if per-unit production subsidy is present or not. Formally we write:

b. $\Delta k^{R I}>\Delta k^{M I}>\Delta k^{R B}>\Delta k^{M B}$.

Where $\Psi_{1}$ is defined in the proof.

The government would always provide a higher per-unit subsidy when it only subsidizes through per-unit production than when it subsidizes through both per-unit production and innovation cost (i.e., $\mathrm{t}^{\mathrm{XP}}>\mathrm{t}^{\mathrm{xB}}$ ). This is because the government can spread the subsidy when utilizing both forms of subsidy, while it focuses all subsidy into per-unit production when it only uses a single type of subsidy.

Moreover, innovation subsidy is always larger when the retailer initiates a $R J V\left(\Delta k^{R y}>\right.$ $\left.\Delta k^{M y}\right)$. In contrast, the tax credit parameter is (weakly) larger when the manufacturer initiates $\left(t^{M y} \geq t^{R y}\right)$ for costly innovation $\left(k>\Psi_{1}\right)$. This is because when innovation is not costly ( $k$ is small), the fraction of cost/subsidy $(\lambda)$ that the manufacturer is responsible for would be relatively invariant to whether the collaboration is initiated by the manufacturer or by the retailer. However, as shown in Lemma 1b, when innovation becomes more costly, the collaboration level increases when the retailer initiates collaboration, while the collaboration level decreases 
otherwise. As a result, when innovation is costly ( $k$ is large), the manufacturer's collaboration level would be much larger ( $\lambda$ large) if the project is initiated by the retailer than if it is initiated by the manufacturer. Then the government would encourage more demand by increasing the perunit tax subsidy, $t$, when the manufacturer initiates the RJV because the retailer has a high level of involvement and would set a retail price that best aligns with the government's incentive. Note that the value of $\Psi_{1}$ is a function of the marginal benefit in effort level to government's objective function $(\alpha)$ and the impact of effort to the per-unit production cost $(\beta) . \Psi_{1}$ decreases in $\beta$ implying that the per-unit tax subsidy under system MP is larger than that under system RP, when sustainability innovation is costly ( $k$ large).

As such, the relationship between different values of $t$ may not be intuitively clear. In Figure 7, we visualize the relationship between different $t$ values, and show how $t^{R P}$ and $t^{M P}$ change with the model parameters when $\alpha=0.5$ or $\alpha=1$. The all-white region is the infeasible region where the problem is ill defined — that is, SOC conditions or individual rationality is not satisfied for the supply chain partners. For any fixed value of $k$, the light grey region (the scenario where $\left.t^{R P}>t^{M P}\right)$ is always below the dark grey region $\left(t^{R P}<t^{M P}\right)$, implying that for small per-unit cost increase, the tax credit parameter is larger when the retailer initiates the collaboration and the government uses per-unit subsidy. 

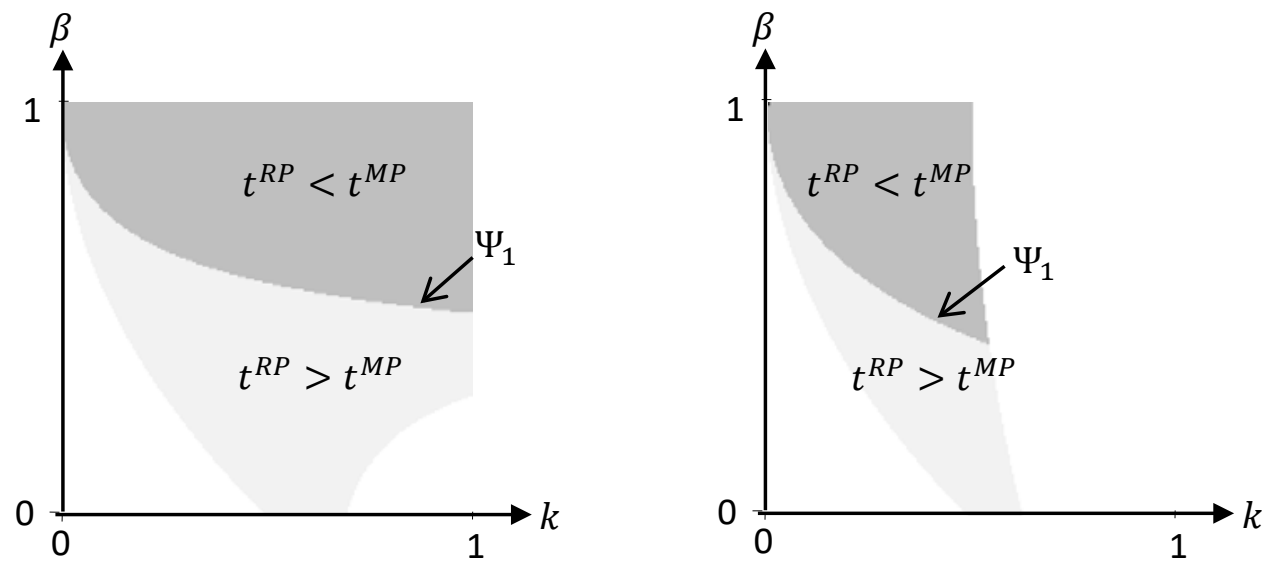

Figure 7: Comparison of $t^{R P}$ and $t^{M P}$ when $\alpha=0.5$ (left) and when $\alpha=1$ (right)

For any given supply chain RJV structure, Proposition B1b shows that the subsidy parameter is always smaller when the government uses both kinds of subsidy than when it only uses one type of subsidy $\left(t^{x B}<t^{x P}\right.$ and $\left.\Delta k^{x B}<\Delta k^{x I}\right)$. This is because when the government uses two different forms of subsidy, it can better incentivize the firms to collaborate and innovate at the desired level.

\section{Appendix C: Proof of Propositions}

\section{Lemma 1 (Sensitivity Analysis):}

a. $\frac{\partial t^{x y}}{\partial k}<0 ; \frac{\partial t^{x y}}{\partial \beta}<0 ; \frac{\partial \Delta k^{x y}}{\partial k}>0 ; \frac{\partial \Delta k^{x y}}{\partial \beta}>0$.

b. $\frac{\partial \lambda^{R y}}{\partial k}>0 ; \frac{\partial \lambda^{R y}}{\partial \beta}>0 ; \frac{\partial \lambda^{M y}}{\partial k}<0 ; \frac{\partial \lambda^{M y}}{\partial \beta}<0$.

c. $\frac{\partial e^{x y}}{\partial k}<0 ; \frac{\partial e^{x y}}{\partial \beta}<0$

\section{Proof of Lemma 1:}

Recall from the derivation of equilibrium (cf. Appendix A) that $\Gamma_{x y}>0$. Moreover, $k>\frac{(1-\beta)^{2}}{2}$ is necessary to satisfy all SOCs, $\beta<1$ is the non-trivial region where effort is non-negative, and 
$p^{R B}=\frac{(1+\alpha-\beta) \beta}{\Gamma 13}>0 \Leftrightarrow \beta>0$. Therefore, we consider the region where $\alpha>0, k>\frac{(1-\beta)^{2}}{2}$ and $0<\beta<1$.

Part a:

(i) $\frac{\partial t^{R P}}{\partial k}=\frac{128(1-\beta)\left(-256(3+4 \alpha-3 \beta) k^{2}+32(7+12 \alpha-7 \beta)(1-\beta)^{2} k-(19+52 \alpha-19 \beta)(1-\beta)^{4}\right)}{\Gamma_{\mathrm{RP}}^{2}}$. Note that the discriminant of the numerator's quadratic polynomial as a function of $k$ is -8192 (1$\beta)^{4}\left(8 \alpha^{2}+8 \alpha(1-\beta)+(1-\beta)^{2}\right)<0$. Therefore, there is no root to $\frac{\partial t^{R P}}{\partial k}=0$, so $\frac{\partial t^{R P}}{\partial k}$ would never change sign. Consequently, it can easily be shown that $\frac{\partial t^{R P}}{\partial k}<0$. We use a similar technique to show that $\frac{\partial t^{M P}}{\partial k}<0$, and the details are omitted to avoid redundancy. Lastly, note that $t^{R B}=t^{M B}$ and $\frac{\partial t^{R B}}{\partial k}=\frac{-6(1-\beta)(1+\alpha-\beta)}{\Gamma_{\mathrm{RB}}^{2}}<0$.

(ii) $\frac{\partial t^{R P}}{\partial \beta}=\frac{-32 \Psi_{\mathrm{t}_{R P}}}{\Gamma_{\mathrm{RP}}}$, where $\Psi_{\mathrm{t}_{R P}}=2048(3+2 \alpha-3 \beta) k^{3}-256(1-\beta)^{2}(7-\alpha-7 \beta) k^{2}-$ $8(1-\beta)^{4}(42 \alpha-19+19 \beta) k+51 \alpha(1-\beta)^{6}$. We use the method proposed by Pun (2015) to show that $\Psi_{\mathrm{t}_{R P}}>0$ for all $\alpha>0, k>\frac{(1-\beta)^{2}}{2}$, and $0<\beta<1$. Specifically, $\Psi_{\mathrm{t}_{R P}}$ is a polynomial, which is continuous and differentiable, so the minimum is either at the solution of the FOCs $\frac{\partial \Psi_{\mathrm{t}_{R P}}}{\partial \alpha}=0, \frac{\partial \Psi_{\mathrm{t}_{R P}}}{\partial k}=0$, and $\frac{\partial \Psi_{\mathrm{t}_{R P}}}{\partial \beta}=0$, or at the boundary. Since there is no solution to these FOCs in the domain, the minimum of $\Psi_{\mathrm{t}_{R P}}$ is at the four boundaries. [Case $1-\alpha=0$ ]: At this boundary, $\Psi_{\mathrm{t}_{R P}}^{\alpha=0}=6144 k^{3}(1-\beta)-1792 k^{2}(1-\beta)^{3}+152 k(1-\beta)^{5}$. There is no solution to the FOCs $\frac{\partial \Psi_{\mathrm{t}_{R P}}^{\alpha=0}}{\partial k}=0$ and $\frac{\partial \Psi_{\mathrm{t}_{R P}}^{\alpha=0}}{\partial \beta}=0$ in the domain $k>\frac{(1-\beta)^{2}}{2}$ and $0<\beta<1$. Moreover, $\Psi_{\mathrm{t}_{R P}}^{\alpha=0}=396(1-\beta)^{7}$ when $k=\frac{(1-\beta)^{2}}{2}, \Psi_{\mathrm{t}_{R P}}^{\alpha=0}=0$ when $\beta=1$, and $\Psi_{\mathrm{t}_{R P}}^{\alpha=0}=8 k(19-224 k+$ $768 k^{2}$ ) when $\beta=0$, which can be shown to be positive. Since $\Psi_{\mathrm{t}_{R P}=0}^{\alpha=0}>0$ when $k=1$ and $\beta=$ $0.5, \Psi_{\mathrm{t}_{R P}}^{\alpha=0}>0$ for all $k>\frac{(1-\beta)^{2}}{2}$ and $0<\beta<1$. [Case $2-k=\frac{(1-\beta)^{2}}{2}$ ]: $\Psi_{\mathrm{t}_{R P}}^{k=\frac{(1-\beta)^{2}}{2}}=$ $459 \alpha(1-\beta)^{6}+396(1-\beta)^{7}>0$. [Case $3-\beta=1$ ]: $\Psi_{\mathrm{t}_{R P}}^{\beta=1}=4096 k^{3} \alpha>0$. [Case $4-\beta=0$ ]: $\Psi_{\mathrm{t}_{R P}}^{\beta=0}=2048 k^{3}(3+2 \alpha)-256 k^{2}(7-\alpha)-8 k(42 \alpha-19)+51 \alpha$. There is no solution to the FOCs $\frac{\partial \Psi_{\mathrm{t}_{R P}}^{\beta=0}}{\partial k}=0$ and $\frac{\partial \Psi_{\mathrm{t}_{R P}}^{\beta=0}}{\partial \beta}=0$ in the domain $k>\frac{1}{2}$ and $\alpha>0$. Moreover, $\Psi_{\mathrm{t}_{R P}}^{\beta=0}=9(44+$ 
$51 \alpha$ ) when $k=\frac{1}{2}$ and $\Psi_{\mathrm{t}_{R P}}^{\beta=0}=8 k\left(19-224 k+768 k^{2}\right)>0$ when $\alpha=0$, so $\Psi_{\mathrm{t}_{R P}}^{\beta=0}>0$. In conclusion, since there is no solution to the FOCs of $\Psi_{t_{R P}}$ and $\Psi_{t_{R P}}>0$ at the boundaries, $\Psi_{\mathrm{t}_{\mathrm{RP}}}>0$ is true for all $\alpha>0, k>\frac{(1-\beta)^{2}}{2}$, and $0<\beta<1$. As a result, $\frac{\partial t^{R P}}{\partial \beta}<0$. We use a similar technique to show that $\frac{\partial t^{M P}}{\partial \beta}<0$, and the details are omitted to avoid redundancy. Lastly, note that $t^{R B}=t^{M B}$ and $\frac{\partial t^{R B}}{\partial \beta}=\frac{-3\left(2 k(2+\alpha-2 \beta)+\alpha(1-\beta)^{2}\right)}{\Gamma_{\mathrm{RB}}^{2}}<0$.

(iii) $\frac{\partial \Delta k^{R I}}{\partial k}=\frac{3+16 \alpha-3 \beta}{7+16 \alpha-7 \beta}>0, \frac{\partial \Delta k^{R B}}{\partial k}=\frac{\alpha}{1+\alpha-\beta}>0, \frac{\partial \Delta k^{M I}}{\partial k}=\frac{1+16 \alpha-\beta}{7+16 \alpha-7 \beta}>0, \frac{\partial \Delta k^{M B}}{\partial k}=\frac{2 \alpha+\beta-1}{2(1+\alpha-\beta)}>$ $0 \Leftrightarrow 2 \alpha+\beta>1$, which is true because $2 \alpha+\beta>1$ is a necessary condition for $\Delta k^{M B}>0$.

(iv) $\frac{\partial \Delta k^{R I}}{\partial \beta}=\frac{512 k \alpha+(1-\beta)\left(768 \alpha^{2}+336 \alpha(1-\beta)+49(1-\beta)^{2}\right)}{8(7+16 \alpha-7 \beta)^{2}}>0, \frac{\partial \Delta k^{R B}}{\partial \beta}=\frac{8 k \alpha+(1+3 \alpha-\beta)^{2}(1-\beta)}{8(1+\alpha-\beta)^{2}}>0$, $\frac{\partial \Delta k^{M I}}{\partial \beta}=\frac{3 \alpha(32 k+(7+32 \alpha-7 \beta)(1-\beta))}{(7+16 \alpha-7 \beta)^{2}}>0, \frac{\partial \Delta k^{M B}}{\partial \beta}=\frac{3 \alpha(2 k+(1+2 \alpha-\beta)(1-\beta))}{4(1+\alpha-\beta)^{2}}>0$.

Part b:

(i) $\frac{\partial \lambda^{R P}}{\partial k}=\frac{(1-\beta)^{2}}{16 k^{2}}>0, \frac{\partial \lambda^{R I}}{\partial k}=\frac{64(7+16 \alpha-7 \beta)(1-\beta)}{(64 k+(7+48 \alpha-7 \beta)(1-\beta))^{2}}>0, \frac{\partial \lambda^{R B}}{\partial k}=\frac{16(1-\beta)(1+\alpha-\beta)}{(16 k+(1+9 \alpha-\beta)(1-\beta))^{2}}>0$.

(ii) $\frac{\partial \lambda^{R P}}{\partial \beta}=\frac{1-\beta}{8 k}>0, \frac{\partial \lambda^{R I}}{\partial \beta}=\frac{32\left(4 k(7+8 \alpha-7 \beta)+7 \alpha(1-\beta)^{2}\right)}{(64 k+(7+48 \alpha-7 \beta)(1-\beta))^{2}}>0, \frac{\partial \lambda^{R B}}{\partial \beta}=\frac{8\left(2 k(2+\alpha-2 \beta)+\alpha(1-\beta)^{2}\right)}{(16 k+(1+9 \alpha-\beta)(1-\beta))^{2}}>0$.

(iii) $\frac{\partial \lambda^{M P}}{\partial k}=\frac{-16}{9(1-\beta)^{2}}<0, \frac{\partial \lambda^{M I}}{\partial k}=\frac{-32}{3(7+16 \alpha-7 \beta)(1-\beta)}<0, \frac{\partial \lambda^{M B}}{\partial k}=\frac{-8}{3(1-\beta)(1+\alpha-\beta)}<0$.

(iv) $\frac{\partial \lambda^{M P}}{\partial \beta}=\frac{-32 k}{9(1-\beta)^{3}}<0, \frac{\partial \lambda^{M I}}{\partial \beta}=\frac{16\left(4 k(7+8 \alpha-7 \beta)+7 \alpha(1-\beta)^{2}\right)}{-3(7+16 \alpha-7 \beta)^{2}(1-\beta)^{2}}<0, \frac{\partial \lambda^{M B}}{\partial \beta}=\frac{4\left(2 k(2+\alpha-2 \beta)+\alpha(1-\beta)^{2}\right)}{-3(1+\alpha-\beta)^{2}(1-\beta)^{2}}<$

0 .

Part c:

Comparing the solutions shows that $e^{R I}=e^{M I}$ and $e^{R B}=e^{M B}$. Then (i) $\frac{\partial e^{R P}}{\partial k}=$ $\frac{-128(1-\beta)\left(256 k^{2}+(7+80 \alpha-7 \beta)(-1+\beta)^{3}-32 k(-1+\beta)(-1+8 \alpha+\beta)\right)}{\Gamma_{R P}^{2}}$. Using the method presented in Part a, the numerator is negative. $\frac{\partial e^{M P}}{\partial k}=\frac{-3(1-\beta)\left(32 k^{2}-48 k \alpha(-1+\beta)+9 \alpha(-1+\beta)^{3}\right)}{2 k^{2} \Gamma_{M P}^{2}}<0 \quad ; \quad \frac{\partial e^{x I}}{\partial k}=$ $\frac{-32(7+16 \alpha-7 \beta)}{\Gamma_{x I}^{2}}<0 ; \frac{\partial e^{x B}}{\partial k}=\frac{-2(1+\alpha-\beta)}{\Gamma_{x B}^{2}}<0$

$$
\frac{\partial e^{R P}}{\partial \beta}=\frac{-8\left(4096 k^{3}+256 k^{2}(7+16 \alpha-7 \beta)(1-\beta)-656 k(1-\beta)^{4}+17(1-\beta)^{5}(1-16 \alpha-\beta)\right)}{\Gamma_{R P}^{2}}<0 \quad ; \quad \frac{\partial e^{M P}}{\partial \beta}=
$$

$\frac{6(8 k+3(1+4 \alpha-\beta)(1-\beta))}{-\Gamma_{M P}^{2}}<0 ; \frac{\partial e^{x I}}{\partial \beta}=\frac{7(32 k+(7+32 \alpha-7 \beta)(1-\beta))}{-\Gamma_{x I}^{2}}<0 ; \frac{\partial e^{x B}}{\partial \beta}=\frac{2 k+(1+2 \alpha-\beta)(1-\beta)}{-\Gamma_{x B}^{2}}<0$. 


\section{Proof of Proposition B1:}

Part a: (i) $t^{R P}-t^{R B}=\frac{(1-\beta)\left(16 k-5(1-\beta)^{2}\right)\left(16 k \alpha-(1+9 \alpha-\beta)(1-\beta)^{2}\right)}{\Gamma_{\mathrm{RP}} \Gamma_{\mathrm{RB}}}>0$ because $16 k>5(1-\beta)^{2}$ when $k>(1-\beta)^{2} / 2$ and $\Delta k^{R B}>0 \Leftrightarrow 16 k \alpha>(1+9 \alpha-\beta)(1-\beta)^{2}$.

(ii) $t^{M P}-t^{M B}=\frac{3(1-\beta)\left(4 k-(1-\beta)^{2}\right)\left(2 k(2 \alpha-1+\beta)-3 \alpha(1-\beta)^{2}\right)}{2 k \Gamma_{\mathrm{MP}} \Gamma_{\mathrm{MB}}}>0$ because $4 k>(1-\beta)^{2}$ when $k>$ $(1-\beta)^{2} / 2$ and $\Delta k^{M B}>0 \Leftrightarrow 2 k(2 \alpha-1+\beta)>3 \alpha(1-\beta)^{2}$.

(iii) Comparing the equilibrium solution shows that $t^{R B}=t^{M B}$.

(iv) $\quad t^{M P}-t^{R P}=\frac{(1-\beta)\left(16 k-3(1-\beta)^{2}\right) \Gamma_{t M P t R P}}{2 k \Gamma_{\mathrm{RP}} \Gamma_{\mathrm{MP}}} \quad$, where $\quad \Gamma_{t M P t R P}=64(4 \alpha-3+3 \beta) k^{2}-$ $4(1-\beta)^{2}(72 \alpha-11+11 \beta) k+51 \alpha(1-\beta)^{4}$. Note that $16 k-3(1-\beta)^{2}>0$ when $k>$ $(1-\beta)^{2} / 2$. There are two solutions to $\Gamma_{t M P t R P}=0$, but the smaller root is smaller than $(1-\beta)^{2} / 2$, so $t^{M P}>t^{R P} \Leftrightarrow k>\Psi_{1}=\frac{72 \alpha(1-\beta)^{2}-11(1-\beta)^{3}+(1-\beta)^{2} \sqrt{1920 \alpha^{2}+864 \alpha(1-\beta)+121(1-\beta)^{2}}}{32(4 \alpha-3+3 \beta)}$.

To show that the solution space is not empty, note that if $\alpha=1$ and $\beta=\frac{1}{2}$, then $t^{M P}<t^{R P}$ when $k=0.35$ and $t^{M P}>t^{R P}$ when $k=0.4$.

Part $\quad$ b: $\quad \Delta k^{R I}-\Delta k^{M I}=\frac{(1-\beta) \Gamma_{\mathrm{RI}}}{16(16 \alpha+7-7 \beta)}>0 \quad, \quad \Delta k^{M I}-\Delta k^{R B}=$ $\frac{(1-\beta)\left(16 k(1+10 \alpha-\beta)+(1-\beta)\left(96 \alpha^{2}+31 \alpha(1-\beta)+7(1-\beta)^{2}\right)\right)}{16(16 \alpha+7-7 \beta)(1+\alpha-\beta)}>0 \quad \quad \quad \quad \quad \quad \quad k^{R B}-\Delta k^{M B}=$ $\frac{(1-\beta)(8 k+(1-\beta)(3 \alpha-1+\beta))}{16(1+\alpha-\beta)}>0$ because $8 k+(1-\beta)(3 \alpha-1+\beta)>0$ when $k>(1-\beta)^{2} / 2$.

\section{Proof of Proposition 1:}

Part a:

$$
\lambda^{R P}-\lambda^{M P}=\frac{\left(16 k-3(1-\beta)^{2}\right)\left(16 k+3(1-\beta)^{2}\right)}{144 k(1-\beta)^{2}}>0 \quad, \quad \lambda^{R I}-\lambda^{M I}=
$$

$\frac{\left(32 k-7(1-\beta)^{2}\right)(64 k+(80 \alpha+21-21 \beta)(1-\beta))}{3(1-\beta)(16 \alpha+7-7 \beta)(64 k+(48 \alpha+7-7 \beta)(1-\beta))}>0$
$\frac{(8 k+(1-\beta)(3 \alpha-1+\beta))(16 k+(3+11 \alpha-3 \beta)(1-\beta))}{3(1-\beta)(1+\alpha-\beta)(16 k+(1+9 \alpha-\beta)(1-\beta))}>0$.

(ii) $e^{M P}-e^{R P}=\frac{(1-\beta)\left(16 k-3(1-\beta)^{2}\right) \Gamma_{\mathrm{e}^{\mathrm{MP}} e^{R P}}}{2 k \Gamma_{\mathrm{RP}} \Gamma_{\mathrm{MP}}}$, where $\Gamma_{\mathrm{e}^{\mathrm{MP}}} e^{R P}=64 k^{2}-51 \alpha(1-\beta)^{3}+4 k(1-$ $\beta)(-13+20 \alpha+13 \beta)$. Note that $16 k-3(1-\beta)^{2}>0$ when $k>(1-\beta)^{2} / 2$. There are two 
solutions to $\Gamma_{\mathrm{e}} \mathrm{MP} e^{R P}=0$, but the smaller root is smaller than $(1-\beta)^{2} / 2$, so $e^{R P}<e^{M P} \Leftrightarrow k>$ $\Psi_{e 1}=\frac{1-\beta}{32}\left(-20 \alpha+13(1-\beta)+\sqrt{169+296 \alpha+400 \alpha^{2}-338 \beta-296 \alpha \beta+169 \beta^{2}}\right)$. То

show that the solution space is not empty, note that if $\alpha=1$ and $\beta=\frac{1}{2}$, then $e^{R P}>e^{M P}$ when $k=0.15$ and $e^{R P}<e^{M P}$ when $k=0.2$.

Part b:

(i) $\quad \lambda^{R P}-\lambda^{R B}=\frac{(1-\beta)\left(16 k \alpha-(1+9 \alpha-\beta)(1-\beta)^{2}\right)}{16 k(16 k+(1+9 \alpha-\beta)(1-\beta))}>0$ because $\quad \Delta k^{R B}>0 \Leftrightarrow 16 k \alpha>(1+9 \alpha-$ $\beta)(1-\beta)^{2} \cdot \lambda^{R B}-\lambda^{R I}=\frac{24(1-\beta)(1+4 \alpha-\beta)(2 k+\alpha-\alpha \beta)}{(16 k+(1+9 \alpha-\beta)(1-\beta))(64 k+(7+48 \alpha-7 \beta)(1-\beta))}>0$.

(ii) $e^{R B}-e^{R P}=\frac{\left(16 k \alpha-(1+9 \alpha-\beta)(1-\beta)^{2}\right)\left(16 k-9(1-\beta)^{2}\right)}{\Gamma_{\mathrm{RP}} \Gamma_{\mathrm{RB}}}>0$ because $\quad$ (a) $\Delta k^{R B}>0 \Leftrightarrow 16 k \alpha-$ $(1+9 \alpha-\beta)(1-\beta)^{2}>0$ and (b) $\Delta k^{R B}>0 \Leftrightarrow \alpha\left(16 k-9(1-\beta)^{2}\right)>(1-\beta)^{3} \Rightarrow 16 k-$ $9(1-\beta)^{2}>0 \quad$. $\quad e^{R B}-e^{R I}=\frac{9(1-\beta)(2 k+\alpha-\alpha \beta)}{\Gamma_{\mathrm{RI}} \Gamma_{\mathrm{RB}}}>0 \quad . \quad e^{R I}-e^{R P}=$ $\frac{256 k^{2}(-9+16 \alpha+9 \beta)-32 k(1-\beta)^{2}(-1+144 \alpha+\beta)+9(7+80 \alpha-7 \beta)(1-\beta)^{4}}{\Gamma_{\mathrm{RP}} \Gamma_{\mathrm{RI}}}$. There are two roots to the numerators, but the smaller root is smaller than $(1-\beta)^{2} / 2$, so $e^{R P}<e^{R I} \Leftrightarrow k>\Psi_{e 2}=$ $\frac{144 \alpha-(1-\beta)+2 \sqrt{2} \sqrt{71+648 \alpha+1152 \alpha^{2}-142 \beta-648 \alpha \beta+71 \beta^{2}}}{16(-9+16 \alpha+9 \beta)}(1-\beta)^{2}$. To show that the solution space is not empty, note that if $\alpha=1$ and $\beta=\frac{1}{2}$, then $e^{R P}>e^{R I}$ when $k=0.2$ and $e^{R P}<e^{R I}$ when $k=$ 0.5 .

Part c:

(i) $\lambda^{M I}-\lambda^{M B}=\frac{4(4 \alpha+1-\beta)(2 k+\alpha-\alpha \beta)}{(16 \alpha+7-7 \beta)(1+\alpha-\beta)(1-\beta)}>0 . \lambda^{M B}-\lambda^{M P}=\frac{4\left(2 k(2 \alpha-1+\beta)-3 \alpha(1-\beta)^{2}\right)}{9(1-\beta)^{2}(1+\alpha-\beta)}>0$ because $\Delta k^{M B}>0 \Leftrightarrow 2 k(2 \alpha-1+\beta)>3 \alpha(1-\beta)^{2}$.

(ii) $\quad e^{M B}-e^{M P}=\frac{\left(4 k-3(1-\beta)^{2}\right)\left(2 k(-1+2 \alpha+\beta)-3 \alpha(1-\beta)^{2}\right)}{2 k \Gamma_{\mathrm{MP}} \Gamma_{\mathrm{MB}}}>0 \quad$ because $\quad$ (a) $\quad \Delta k^{M B}>0 \Leftrightarrow$ $2 k(-1+2 \alpha+\beta)-3 \alpha(1-\beta)^{2}>0 \quad$ and $\quad$ (b) $\quad \Delta k^{M B}>0 \Leftrightarrow \alpha\left(4 k-3(1-\beta)^{2}\right)>$ $2 k(1-\beta) \Rightarrow 4 k-3(1-\beta)^{2}>0 \quad \cdot \quad e^{M B}-e^{M I}=\frac{9(1-\beta)(2 k+\alpha-\alpha \beta)}{\Gamma_{\mathrm{MI}} \Gamma_{\mathrm{MB}}}>0 \quad \cdot \quad e^{M I}-e^{M P}=$ $\frac{16 k^{2}(-17+16 \alpha+17 \beta)-6 k(1-\beta)^{2}(-7+64 \alpha+7 \beta)+63 \alpha(1-\beta)^{4}}{2 k \Gamma_{\mathrm{MP}} \Gamma_{\mathrm{MI}}}$. There are two roots to the numerators, but the smaller root is smaller than $(1-\beta)^{2} / 2$, so $e^{M P}<e^{M I} \Leftrightarrow k>\Psi_{e 3}=$ $\frac{3\left(64 \alpha-7(1-\beta)+\sqrt{49+1008 \alpha+2304 \alpha^{2}-98 \beta-1008 \alpha \beta+49 \beta^{2}}\right)}{16(-17+16 \alpha+17 \beta)}(1-\beta)^{2}$. To show that the solution space is 
not empty, note that if $\alpha=1$ and $\beta=\frac{1}{2}$, then $e^{M P}>e^{M I}$ when $k=0.5$ and $e^{M P}<e^{M I}$ when $k=0.8$.

\section{Proof of Proposition 2:}

$\pi_{M}^{R P}-\pi_{M}^{R B}=\frac{(1-\beta)\left(16 k \alpha-(1+9 \alpha-\beta)(1-\beta)^{2}\right) \Psi_{\pi_{M}^{R P} \pi_{M}^{R B}}}{2 \Gamma_{\mathrm{RP}}^{2} \Gamma_{\mathrm{RB}}^{2}}, \quad$ where $\quad \Psi_{\pi_{M}^{R P} \pi_{M}^{R B}}=8192 k^{4}+512 k^{3}(1-$ $\beta)(-11+8 \alpha+11 \beta)-32 k^{2}(1-\beta)^{3}(-19+88 \alpha+19 \beta)+2 k(55+184 \alpha-55 \beta)(1-$ $\beta)^{5}+(1-\beta)^{7}(-12+11 \alpha+12 \beta)$. Using the logic presented in the proof of Lemma 1a(ii), it can be shown that $\Psi_{\pi_{M}^{R P} \pi_{M}^{R B}}>0$. Therefore, $\pi_{M}^{R P}>\pi_{M}^{R B}$ because $\Delta k^{R B}>0 \Leftrightarrow 16 k \alpha-$ $(1+9 \alpha-\beta)(1-\beta)^{2}>0$. We use the same technique to show the remaining inequalities for Parts $a$ and $b$.

Part c: $\pi_{G}^{R B}-\pi_{G}^{R P}=\frac{\left(16 k \alpha-(1+9 \alpha-\beta)(1-\beta)^{2}\right)^{2}}{2 \Gamma_{\mathrm{RP}} \Gamma_{\mathrm{RB}}}>0, \pi_{G}^{R B}-\pi_{G}^{R I}=\frac{9(2 k+\alpha-\alpha \beta)^{2}}{2 \Gamma_{\mathrm{RI}} \Gamma_{\mathrm{RB}}}>0, \pi_{G}^{M B}-\pi_{G}^{M P}=$ $\frac{\left(2 k(-1+2 \alpha+\beta)-3 \alpha(1-\beta)^{2}\right)^{2}}{4 k \Gamma_{\mathrm{MP}} \Gamma_{\mathrm{MB}}}>0, \pi_{G}^{M B}-\pi_{G}^{M I}=\frac{9(2 k+\alpha-\alpha \beta)^{2}}{2 \Gamma_{\mathrm{MI}} \Gamma_{\mathrm{MB}}}>0 . \pi_{G}^{R P}-\pi_{G}^{R I}=\frac{\Psi_{\mathrm{R} 2}}{2 \Gamma_{R P} \Gamma_{R I}}$, where $\Psi_{\mathrm{R} 2}=4608 k^{3}-(1-\beta)^{4}\left(720 \alpha^{2}+126 \alpha(1-\beta)+7(1-\beta)^{2}\right)+2 k(1-\beta)^{2}\left(2304 \alpha^{2}-\right.$ $\left.32 \alpha(1-\beta)+9(1-\beta)^{2}\right)-64 k^{2}\left(64 \alpha^{2}-72 \alpha(1-\beta)+9(1-\beta)^{2}\right)$. This can either be positive or negative. Similarly, $\pi_{G}^{M P}-\pi_{G}^{M I}=\frac{\Psi_{\mathrm{M} 2}}{2 \Gamma_{M P} \Gamma_{M I}}$, where $\Psi_{\mathrm{M} 2}=288 k^{3}-63 \alpha^{2}(1-\beta)^{4}+$ $12 k \alpha(1-\beta)^{2}(-7+32 \alpha+7 \beta)-4 k^{2}\left(64 \alpha^{2}-136 \alpha(1-\beta)+7(1-\beta)^{2}\right)$. This can either be positive or negative. To show that the solution space is not empty, note that if $\alpha=1$ and $k=$ 0.2 , then $\pi_{G}^{x P}>\pi_{G}^{x I}$ when $\beta=0.5$ and $\pi_{G}^{x P}<\pi_{G}^{x I}$ when $\beta=0.8$.

\section{Proof of Proposition 3:}

Part a: (i) $\pi_{M}^{M P}-\pi_{M}^{R P}=\frac{\left(-3+16 k+6 \beta-3 \beta^{2}\right)^{2} \Psi_{2}}{4 k \Gamma_{\mathrm{RP}}^{2} \Gamma_{\mathrm{MP}}^{2}}$, where $\Psi_{2}=65536 k^{5}+4096 k^{4}(1-\beta)(40 \alpha-$ $27(1-\beta))+256 k^{3}(1-\beta)^{2}\left(304 \alpha^{2}-872 \alpha(1-\beta)+171(1-\beta)^{2}\right)-16 k^{2}(1-$ $\beta)^{4}\left(6064 \alpha^{2}-5080 \alpha(1-\beta)+217(1-\beta)^{2}\right)+24 k(1-\beta)^{6}\left(1398 \alpha^{2}-273 \alpha(1-\beta)-\right.$ $\left.(1-\beta)^{2}\right)-2601 \alpha^{2}(1-\beta)^{8}$. Note that $\Psi_{2}$ can either be positive or negative. To show that the solution space is not empty, note that if $\alpha=1$ and $\beta=\frac{1}{2}$, then $\pi_{M}^{M P}<\pi_{M}^{R P}$ when $k=0.17$ and $\pi_{M}^{M P}>\pi_{M}^{R P} \quad$ when $\quad k=0.22$

$$
\pi_{M}^{M I}-\pi_{M}^{R I}=\frac{2 k+\alpha-\alpha \beta}{\Gamma_{\mathrm{MI}}}>0
$$

(iii) $\pi_{M}^{M B}-\pi_{M}^{R B}=$ $\frac{(2 k+\alpha-\alpha \beta)(8 k+(1-\beta)(-1+3 \alpha+\beta))}{4 \Gamma_{\mathrm{MB}}^{2}}>0 \quad$ because $\quad 8 k+(1-\beta)(3 \alpha-1+\beta)>0 \quad$ when $\quad k>$ $(1-\beta)^{2} / 2$. 
Part b: This is true because $\pi_{R}^{M y}=0$.

Part c: (i) $\pi_{G}^{M P}-\pi_{G}^{R P}=\frac{(1-\beta)\left(16 k-3(1-\beta)^{2}\right) \Psi_{\pi_{G}^{R P} \pi_{G}^{M P}}}{4 k \Gamma_{\mathrm{RP}} \Gamma_{\mathrm{MP}}}$, where $\Psi_{\pi_{G}^{R P} \pi_{G}^{M P}}=32 k^{2}(4 \alpha+\beta-1)+$ $2 k(1-\beta)\left(40 \alpha^{2}-52 \alpha(1-\beta)-(1-\beta)^{2}\right)-51 \alpha^{2}(1-\beta)^{3}$. Note that $16 k-3(1-\beta)^{2}>0$ when $k>(1-\beta)^{2} / 2$. We next show the condition for $\Psi_{\pi_{G}^{R P} \pi_{G}^{M P}}>0$. First, there are two roots to $\Psi_{\pi_{G}^{R P} \pi_{G}^{M P}}=0$ when $4 \alpha+\beta-1>0$, but only the larger root $\Psi_{3}=$ $\frac{(1-\beta)\left(-40 \alpha^{2}+52 \alpha(1-\beta)+(1-\beta)^{2}+\sqrt{1600 \alpha^{4}+2368 \alpha^{3}(1-\beta)+992 \alpha^{2}(1-\beta)^{2}+104 \alpha(1-\beta)^{3}+(1-\beta)^{4}}\right)}{32(-1+4 \alpha+\beta)}$ is positive.

Second, when $4 \alpha+\beta-1<0, \Psi_{\pi_{G}^{R P} \pi_{G}^{M P}}<0$ when $k=\frac{(1-\beta)^{2}}{2}$. Moreover, the maximum of $\Psi_{\pi_{G}^{R P} \pi_{G}^{M P}}$ is at $k=\frac{-(1-\beta) \Psi_{x}}{32(1-4 \alpha-\beta)}$, where $\Psi_{x}=1+52 \alpha-40 \alpha^{2}-2 \beta-52 \alpha \beta+\beta^{2}$. Using the method presented in the proof of Lemma 1a(ii), it can be shown that $\Psi_{x}>0$ for all $\alpha>0$ and $0<\beta<1-4 \alpha$. Therefore, $\Psi_{\pi_{G}^{R P} \pi_{G}^{M P}}<0$, so $\pi_{G}^{R P}>\pi_{G}^{M P}$. To show that the solution space is not empty, note that if $\alpha=1$ and $\beta=\frac{1}{2}$, then $\pi_{G}^{M P}<\pi_{M}^{R P}$ when $k=0.17$ and $\pi_{G}^{M P}>\pi_{G}^{R P}$ when $k=0.22$. (ii-iii) The result can be obtained by comparing the equilibrium solution.

\section{Appendix D: Notation}

\begin{tabular}{l|l} 
Term & Meaning \\
\hline$p$ & Product price, set by the retailer \\
& Sustainability effort exerted by the manufacturer \\
& Demand for the product \\
$k$ & The coefficient of effort cost, quadratic in effort (total cost is $k e^{2}$ ) \\
$\beta$ & Per-unit production cost \\
$t$ & Per-unit tax credit for each produced unit \\
$\Delta k$ & The cost of innovation subsidized by the government, net innovation cost is
\end{tabular}




\begin{tabular}{|c|c|}
\hline & $(k-\Delta k) e^{2}$ \\
\hline$\Phi$ & Total subsidies, $\Phi=t D+\Delta k e^{2}$ \\
\hline$\lambda$ & $\begin{array}{l}\text { The fraction of the government subsidy received by the manufacturer; the } \\
\text { remainder, } 1-\lambda \text {, goes to the retailer }\end{array}$ \\
\hline$w$ & Payment from the retailer to the manufacturer (wholesale price/transfer payment) \\
\hline$\alpha$ & Constant marginal benefit of innovation effort for the government \\
\hline$\pi_{M}$ & Manufacturer's profit function \\
\hline$\pi_{R}$ & Retailer's profit function \\
\hline$\pi_{G}$ & Government's profit function \\
\hline
\end{tabular}

\section{References:}

Arora, A., \& Ceccagnoli, M. (2006). Patent Protection, Complementary Assets, and Firms' Incentives for Technology Licensing. Management Science, 52(2), 293-308. http://doi.org/10.1287/mnsc.1050.0437

Atasu, A., Özdemir, Ö., \& Van Wassenhove, L. N. (2013). Stakeholder perspectives on E-waste take-back legislation. Production and Operations Management, 22(2), 382-396. http://doi.org/10.1111/j.1937-5956.2012.01364.x

Atasu, A., \& Subramanian, R. (2012). Extended producer responsibility for e-waste: Individual or collective producer responsibility? Production and Operations Management, 21(6), 1042-1059. http://doi.org/10.1111/j.1937-5956.2012.01327.x

Atasu, A., Van Wassenhove, L. N., \& Sarvary, M. (2009). Efficient Take-Back Legislation. Production and Operations Management, 18(3), 243-258. http://doi.org/10.1111/j.19375956.2009.01004.x

Baker, G. (2014, April). Business \& Environment (A Special Report) --- Wal-Mart’s Green Initiative: Status Report: Chairman Michael Duke on why the retailer's detergent bottles are smaller than they used to be. Wall Street Journal.

Bhaskaran, S. R., \& Krishnan, V. (2009). Effort, Revenue, and Cost Sharing Mechanisms for Collaborative New Product Development. Management Science, 55(7), 1152-1169. http://doi.org/10.1287/mnsc.1090.1010

Canada. (2008). Federal Sustainable Development Act. Retrieved from http://lawslois.justice.gc.ca/eng/acts/F-8.6/ 
Center for Responsive Politics. (2015). Walmart Lobbying Efforts. Retrieved from https://www.opensecrets.org/lobby/clientsum.php?id=D000000367

Cohen, M. C., Lobel, R., \& Perakis, G. (2016). The Impact of Demand Uncertainty on Consumer Subsidies for Green Technology Adoption. Management Science, 62(5), 1235-1258. http://doi.org/10.1287/mnsc.2015.2173

Drake, D. F., Kleindorfer, P. R., \& Van Wassenhove, L. N. (2015). Technology Choice and Capacity Portfolios under Emissions Regulation. Production and Operations Management, 1-34. http://doi.org/10.1111/poms.12523

Drake, D. F., \& Spinler, S. (2013). Sustainable Operations Management: An Enduring Stream or a Passing Fancy? Manufacturing \& Service Operations Management, 15(4), 689-700. http://doi.org/10.1287/msom.2013.0456

Espinoza, J. (2012, September 17). Shedding Light on Subsidies: What incentives exist for renewables? And how exactly do they work? The Wall Street Journal.

European Commission, D.-G. for E. (2013). Attitudes of Europeans towards building the single market for green products. Brussels. Retrieved from http://ec.europa.eu/public_opinion/flash/fl_367_en.pdf

Ge, Z., \& Hu, Q. (2008). Collaboration in R\&D activities: Firm-specific decisions. European Journal of Operational Research, 185(2), 864-883. http://doi.org/10.1016/j.ejor.2007.01.020

Ge, Z., Hu, Q., \& Xia, Y. (2014). Firms’ R\&D Cooperation Behavior in a Supply Chain. Production and Operations Management, 23(4), 599-609. http://doi.org/10.1111/poms.12037

Ghosh, D., \& Shah, J. (2015). Supply chain analysis under green sensitive consumer demand and cost sharing contract. International Journal of Production Economics, 164, 319-329. http://doi.org/10.1016/j.ijpe.2014.11.005

Girotra, K., \& Netessine, S. (2013). OM Forum —Business Model Innovation for Sustainability. Manufacturing \& Service Operations Management, 15(4), 537-544. http://doi.org/10.1287/msom.2013.0451

Global Subsidies Initiative. (2015). Subsidy types. Retrieved September 9, 2015, from https://www.iisd.org/GSI/subsidy-types

InnovateUK. (2015). Innovate UK Overview. Retrieved from https://www.gov.uk/guidance/innovation-apply-for-a-funding-award Accessed on 2/5/2016

Kim, S.-H., \& Netessine, S. (2013). Collaborative Cost Reduction and Component Procurement Under Information Asymmetry. Management Science, 59(1), 189-206.

Kiron, D., Kruschwitz, N., Haanaes, K., \& Reeves, M. (2015). Joining Forces Collaboration and Leadership for Sustainability The growing importance of corporate collaboration and boards of directors to sustainable business. MIT Sloan Management Review. Retrieved from http://flagship.luc.edu/login?url=http://search.ebscohost.com/login.aspx?direct=true\&db=a9 h\&AN=74386489\&site=ehost-live 
Kojoyan, S. (2014). The Economics of Sustainable Coffee Production. Retrieved September 14, 2015, from http://www.triplepundit.com/2014/11/economics-sustainable-coffee-production/

Korn, M. (2008, October). Cleaning Companies Go Green. Wall Street Journal. Retrieved from http://search.proquest.com.proxy.lib.uwaterloo.ca/docview/399022490?accountid=14906

Kraft, T., Zheng, Y., \& Erhun, F. (2013). The NGO’s Dilemma: How to Influence Firms to Replace a Potentially Hazardous Substance. Manufacturing \& Service Operations Management, 15(4), 649-669. http://doi.org/10.1287/msom.2013.0440

Krass, D., Nedorezov, T., \& Ovchinnikov, A. (2013). Environmental taxes and the choice of green technology. Production and Operations Management, 22(5), 1035-1055. http://doi.org/10.1111/poms.12023

Krishnan, V., \& Loch, C. H. (2005). A Retrospective Look at Production and Operations Management Articles on New Product Development. Production and Operations Management, 14(4), 433-441. http://doi.org/10.1007/BF02640937

Krishnan, V., \& Ulrich, K. T. (2001). Product Development Decisions: A Review of the Literature. Management Science, 47(1), 1-21. http://doi.org/10.1287/mnsc.47.1.1.10668

Ministry of Power. (2017). NATIONAL UJALA DASHBOARD | EESL. Retrieved November 19, 2017, from http://www.ujala.gov.in/

Nielsen. (2014). Global Consumers are Willing to Put Their Money Where Their Heart is When it Comes to Goods and Services from Companies Committed to Social Responsibility. Retrieved from http://www.nielsen.com/us/en/press-room/2014/global-consumers-arewilling-to-put-their-money-where-their-heart-is.html

NSW. (2014). Government Resource Efficiency Policy (GREP). Retrieved from http://www.environment.nsw.gov.au/resources/government/140567NSWGREP.pdf

Obama, B. (2015). EO 13514: Planning for Federal Sustainability in the Next Decade. Retrieved from https://www.whitehouse.gov/the-press-office/2015/03/19/executive-order-planningfederal-sustainability-next-decade

Office, U. S. G. A. (1992). Federal Research: SEMATECH's Technological Progress and Proposed R\&amp;D Program. Retrieved from http://www.gao.gov/products/RCED-92223BR

Özdemir, Ö., Denizel, M., \& Guide Jr., V. D. R. (2012). Recovery decisions of a producer in a legislative disposal fee environment. European Journal of Operational Research, 216(2), 293-300. http://doi.org/10.1016/j.ejor.2011.07.035

Palmer, K., \& Walls, M. (1997). Optimal policies for solid waste disposal Taxes, subsidies, and standards. Journal of Public Economics, 65(2), 193-205. http://doi.org/10.1016/S00472727(97)00028-5

Popp, D., Newell, R. G., \& Jaffe, A. B. (2009). Energy, the Environment, and Technological Change (No. 14832). Cambridge, MA, MA. Retrieved from http://www.nber.org/papers/w14832

Raz, G., Druehl, C. T., \& Blass, V. (2013). Design for the environment: Life-cycle approach 
using a newsvendor model. Production and Operations Management, 22(4), 940-957. http://doi.org/10.1111/poms.12011

Raz, G., \& Ovchinnikov, A. (2015). Coordinating Pricing and Supply of Public Interest Goods Using Rebates and Subsidies. IEEE Transactions on Engineering Management, 62(1), 6579.

Rossini, M., Ciarapica, F. E., Matt, D. T., \& Spena, P. R. (2016). A Preliminary Study on the Changes in the Italian Automotive Supply Chain for the Introduction of Electric Vehicles. Journal of Industrial Engineering and Management, 9(2), 450-486. http://doi.org/10.3926/jiem.1504

Srinivasan, L. (2016, January 18). Meet the IIT Madras graduate who has built India’s first solar boat ferry. Daily News and Analysis India. Retrieved from http://www.dnaindia.com/india/report-meet-the-iit-madras-graduate-who-has-built-india-sfirst-solar-boat-ferry-2167266

Talluri, S., Narasimhan, R., \& Chung, W. (2010). Manufacturer cooperation in supplier development under risk. European Journal of Operational Research, 207(1), 165-173. http://doi.org/10.1016/j.ejor.2010.03.041

Tang, C. S., \& Zhou, S. (2012). Research advances in environmentally and socially sustainable operations. European Journal of Operational Research, 223(3), 585-594. http://doi.org/10.1016/j.ejor.2012.07.030

U.S. Congress. (2005). Energy Policy Act of 2005. Retrieved September 9, 2015, from http://www.gpo.gov/fdsys/pkg/PLAW-109publ58/pdf/PLAW-109publ58.pdf

US Congress, \& U.S. Congress. (2009). American Recovery and Reinvestment Act of 2009, Program 1603. Retrieved September 9, 2015, from http://www.gpo.gov/fdsys/pkg/PLAW111publ5/html/PLAW-111publ5.htm

Veugelers, R. (1998). Collaboration in R\&D: An Assessment of Theoretical and Empirical Findings, (3), 419-443. 